\title{
LUT
}

Lappeenranta

University of Technology

\section{New investment decision-making tool that combines a fuzzy inference system with real option analysis}

\author{
Kozlova Mariia, Collan Mikael, Luukka Pasi
}

\author{
This is an Pre-print \\ version of an article \\ published by SIGEF \\ in Fuzzy Economic Review
}

DOI: $\quad 10.25102 / f e r .2018 .01 .04$

Copyright of the original publication: (C) SIGEF 2018

Please cite the publication as follows:

Kozlova, M., Collan, M., Luukka, P. (2018). New investment decision-making tool that combines a fuzzy inference system with real option analysis. Fuzzy Economic Review. Vol 23, Issue 1. p. 63-92. DOI: 10.25102/fer.2018.01.04

This is a parallel published version of an original article. This version can differ from the original published article. 
NEW INVESTMENT DECISION-MAKING TOOL THAT COMBINES A FUZZY INFERENCE SYSTEM WITH REAL OPTION ANALYSIS

\author{
Mariia Kozlova, ${ }^{a}$ Mikael Collan, ${ }^{b}$ and Pasi Luukka ${ }^{\mathrm{c}}$ \\ a,b,c School of Business and Management, Lappeenranta University of \\ Technology
}

Skinnarilankatu 34, 53850 Lappeenranta, Finland

${ }^{a}$ corresponding author, mariia.kozlova@lut.fi, +358 504636168 


\section{NEW INVESTMENT DECISION-MAKING TOOL THAT COMBINES A FUZZY INFERENCE SYSTEM WITH REAL OPTION ANALYSIS}

Abstract: This paper proposes a new procedure for enriching investment and real option analysis performed with the fuzzy pay-off method by decomposing the pay-off distribution into multiple sub-distributions that correspond to different investment scenarios. This creates more information about the importance of the effect of selected factors to investment profitability. Furthermore, based on the proposed procedure, we show how a fuzzy inference system to support investment decision-making can be constructed. The proposed new procedure and the application of a fuzzy inference system are illustrated with a numerical case analysis of a power generation investment.

The results show that the proposed new procedure reveals actionable information about the analyzed investment that may otherwise remain uncovered and enhances the decision-making ability of investment managers. The application of a fuzzy inference system to investment decision-support and real option analysis is a rather new approach. The obtained results highlight how the construct of a fuzzy inference system must be adapted to the perspective of the application for which it is used.

Keywords: Corporate Finance and Governance, Capital Budgeting, Mathematical and Simulation Modeling, Renewable Resources and Conservation 


\section{INTRODUCTION}

Profitability analysis of investments is typically a forward-looking procedure and as such it is ridden with imprecision, connected to the estimated inputs into the analysis process. Profitability analysis has typically been done by using the wellknown net present value, or NPV, method, the most commonly used investment profitability analysis method in the industry (Block, 2007; Graham \& Harvey, 2001; Ryan \& Ryan, 2002). The classical NPV method is, unfortunately, not able to consider estimation imprecision, and nor can it take into consideration the value of flexibility (Block, 2007). Flexibility in investments is often called real optionality. For these reasons and for other reasons that include, e.g., better software and faster computers, simulation-based (Bastian-Pinto, Brandão, \& Hahn, 2009; Boomsma, Meade, \& Fleten, 2012; Cortazar \& Schwartz, 1998; Hacura, Jadamus-Hacura, \& Kocot, 2001; Monjas-Barroso \& Balibrea-Iniesta, 2013; Topal, 2008; Vithayasrichareon \& MacGill, 2012; Zhu, Zhang, \& Fan, 2015) and fuzzy logic-based (Buckley, 1987; Kuchta, 2000; Kuchta, 2001) investment analysis methods are becoming more popular. The resulting output from simulation and fuzzy logic based methods is a profitability distribution, an NPV probability distribution, or a fuzzy number NPV respectively. These results are a clearly different from the single number NPV outcome, provided by the net present value method.

In this paper we focus on fuzzy logic based investment profitability analysis and build on the previously introduced fuzzy pay-off method (FPOM) (Collan, 2012; Collan, Fullér, \& Mezei, 2009). The fuzzy pay-off method is a profitability analysis 
method that deals with cash-flow imprecision by using fuzzy numbers that are typically created from three, or four, cash-flow scenarios. An extension to the payoff method exists for real option valuation (Collan, 2012). The ability to capture cash-flow imprecision and to include the valuation of real options makes the fuzzy pay-off method an interesting addition to the toolkit of investment managers. The pay-off method is a robust method, and thus usable under the condition of structural uncertainty (Collan, Haahtela, \& Kyläheiko, 2016; Kyläheiko, 1998). Previously, the pay-off method has been used for analyzing the profitability of a number of different kinds of investments that range from analyzing patents as investments to the evaluation of fusion energy investments (Bednyagin \& Gnansounou, 2011; Collan \& Kinnunen, 2011; Collan \& Heikkilä, 2011; Collan, 2011; Hassanzadeh, Collan, \& Modarres, 2012b; Hassanzadeh, Collan, \& Modarres, 2012a; Kozlova, Collan, \& Luukka, 2015).

In this paper, by using the pay-off method as a basis, we introduce a new procedure that extends the fuzzy pay-off method, and that can be used to create more insight for the decision-maker, when the fuzzy NPV outcome created by the pay-off method is a composition of multiple variables. The idea of this new procedure is to enable the decomposition of the, from the pay-off method resulting, fuzzy NPV into pieces, or fuzzy sub-NPVs (sub-distributions) that are matched to the variable state combinations that cause them. In other words, a connection between the cause and the effect is established. This kind of information is much more actionable, than only having access to the resulting fuzzy NPV. Understanding what causes the positive and / or negative NPV 
outcomes is actionable information and allows managers to act, in order to steer the situation towards a good, positive NPV. A similar procedure for simulationbased profitability analysis has been introduced in (Kozlova, Collan, \& Luukka, 2016a). We call this new procedure "fuzzy pay-off distribution decomposition".

Additionally, in this paper, we show how a fuzzy inference system (FIS) can be constructed for the purposes of supporting and even automating investment decision-making. Constructing a fuzzy inference system "on top" of the procedure used in the fuzzy pay-off distribution decomposition quite simple as the input and output components to the are already defined.

We illustrate how the new fuzzy pay-off decomposition procedure works with a profitability analysis case of a solar photovoltaic power generation investment. Furthermore, we show how a fuzzy inference system for decision-making "automation" can be constructed, based on the same case.

The rest of this paper is structured as follows: first, we briefly present the underlying concepts of the fuzzy pay-off method and fuzzy inference system. This is followed by the introduction of the proposed new fuzzy pay-off distribution decomposition procedure and a presentation of the architecture of a fuzzy inference system. The proposed new procedure and the application of a FIS are illustrated with a numerical case. We demonstrate how the fuzzy inference system can be used for policy analysis and as a support for investment decisionmaking. The paper is closed with a discussion and conclusions. 


\section{BACKGROUND}

In this section, we briefly introduce the two important concepts that lie behind the new fuzzy pay-off distribution decomposition procedure: the fuzzy pay-off method and the Mamdani-type fuzzy inference system.

\subsection{FUZZY PAY-OFF METHOD}

The fuzzy pay-off method (FPOM) (Collan et al., 2009) is a modern technique to value investment projects in the presence of uncertainty and imprecise cashflows. The method is based on using expert estimated cash-flow scenarios for an investment as a basis for the analysis. Typically, the experts are asked to estimate three, or four, cash-flow scenarios that correspond to the minimum possible, the maximum possible, and a best estimate scenario. A net present value (Brealey, Myers, Allen, \& Mohanty, 2012) is calculated for each one of these scenarios and the resulting NPVs are then used to create a pay-off distribution for the investment. The pay-ff distribution is treated as a fuzzy number, a fuzzy net present value distribution. Real option value for the investment can be calculated directly from the pay-off distribution. The procedure that is the FPOM for a three scenario case can be shortly described as follows:

1. Estimation of three future project cash-flow scenarios: the minimum possible, the best estimate, and the maximum possible scenarios. If it is necessary the cash-flow scenarios may consist of separate "sub" scenarios representing various classes of costs and revenues; 
2. NPV calculation for each cash-flow scenario. Discussion on linking cash-flow sub-scenarios within the context of NPV calculation is discussed, e.g., in (Collan, 2012);

3. Construction of a triangular fuzzy pay-off (NPV) distribution for the project: the minimum possible and the maximum possible scenario NPVs are assigned a limit to zero degree of membership in the set of possible pay-offs. The distribution is assumed to be bounded and the minimum and the maximum possible values form the lower and the upper limits of the distribution. The best estimate scenario NPV is considered to fully belong to the set of possible pay-offs and is assigned full membership. The relationship between the limits of the distribution and the best estimate scenario value is assumed to be linear and hence the pay-off distribution is assumed to be of a triangular shape. The created triangular pay-off distribution is treated as a fuzzy number. The membership function for the triangular pay-off distribution can be defined as:

$$
\mu_{N P V}(x ; \alpha, \beta, \gamma)=\left\{\begin{array}{c}
\frac{x-\alpha}{\beta-\alpha} \text { if } \alpha \leq x \leq \beta \\
\frac{\gamma-x}{\gamma-\beta} \text { if } \beta<x \leq \gamma \\
0 \text { otherwise }
\end{array}\right.
$$

where $\alpha$ is the minimum possible scenario NPV,

$\beta$ is the best estimate scenario NPV, and $y$ is the maximum possible scenario NPV. 
4. Computing the real option value. The real option value is defined as the possibilistic mean of the positive part of the distribution weighted on the success-ratio (the area over positive NPV part of the distribution / total area of the distribution):

$\mathrm{RO}$ value $=$ possibilistic mean of the positive area * positive area / whole area In the original version of the FPOM (Collan et al., 2009), the mean value is computed as a possibilistic mean, however, calculating it as the center of gravity is also possible (Borges \& Meier, 2017; Luukka \& Collan, 2015a).

So far, the fuzzy pay-off method has been applied to a number of real-word problems that, among others, include $R \& D$ project selection (Bednyagin \& Gnansounou, 2011; Hassanzadeh et al., 2012b), analysis of large industrial investments (Collan, 2011), analysis of real estate investments (Vimpari, Kajander, \& Junnila, 2014; Vimpari \& Junnila, 2015), patent valuation (Collan \& Heikkilä, 2011; Collan, Fedrizzi, \& Luukka, 2013; Collan \& Kyläheiko, 2013), insurance pricing (Collan, Fedrizzi, \& Luukka, 2016; Luukka \& Collan, 2015b), and M\&A target screening (Collan \& Kinnunen, 2011). The method has also been comparatively analyzed with other methods in (Favato, Cottingham, \& Isachenkova, 2015; Kozlova, Collan, \& Luukka, 2016b).

\subsection{FUZZY INFERENCE SYSTEM}

A fuzzy inference system (FIS) is a system that maps (combinations of) inputs to outputs by applying the fuzzy set theory. Typically the creation of a FIS includes the formulation of a set of rules, or a rule system, that describes how an output is 
inferred from given fuzzy inputs. Perhaps the most commonly used FIS is the Mamdani-type fuzzy inference system (Mamdani \& Assilian, 1975), which is also used here. The FIS used in this research is composed of four main components, which are given below and also illustrated in Figure 1.

1. A rule base: consisting of a number of if-then rules. These rules are typically a quantifiable reasoning scheme that has been created on the basis of linguistic descriptions from experts.

2. Fuzzy inference engine: mimics expert decision-making by "interpreting" the input information with the knowledge derived from the rule-base.

3. Interface for matching crisp estimates with fuzzy input scales: converts inputs coming from an expert into information that is usable by the inference mechanism. This component is sometimes called a "fuzzification interface".

4. Interface for defuzzification: fuzzy result from the inference mechanism are converted into single number outputs.

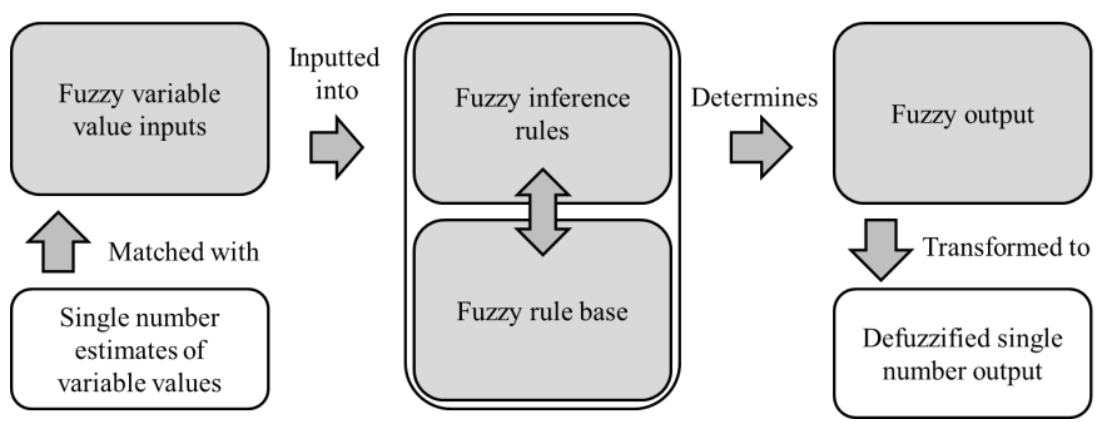

Figure 1. General structure of the fuzzy inference system used 
The use of a fuzzy inference systems is commonplace in a wide variety of application areas that include, e.g., control (Chiou, Chiou, Chu, \& Lin, 2009; CUI, TAN, AO, \& KANG, 2005; Das \& Kar, 2006; Fazzolari, Alcala, Nojima, Ishibuchi, \& Herrera, 2013; Feng, 2006; Tanaka \& Sano, 1994), forecasting in technical and natural systems (Aggarwal, Kumar, Saini, \& Kumar, 2011; Al-zahra, Moosa, \& Jasim, 2015; Bacanli, Firat, \& Dikbas, 2009; Kazeminezhad, Etemad-Shahidi, \& Mousavi, 2005; Mohandes, Rehman, \& Rahman, 2011; Sachdeva, Singh, Singh, \& Arora, 2011; Ying \& Pan, 2008), stock market return prediction (Chang \& Liu, 2008; Esfahanipour \& Aghamiri, 2010; Patel \& Marwala, 2006), and decisionmaking modeling (Amindoust, Ahmed, Saghafinia, \& Bahreininejad, 2012; Ganga \& Carpinetti, 2011; Oderanti \& De Wilde, 2010; Oh, Yang, \& Lee, 2012; Osiro, Lima-Junior, \& Carpinetti, 2014; Yang, Khan, \& Sadiq, 2011). Previously FIS has been used in connection with project valuation in (Ustundag, Kılınç, \& Cevikcan, 2010), where a FIS was used for input parameter definition, and in conjunction with system dynamic modeling for real option valuation in (Arasteh \& Aliahmadi, 2014). Generally speaking, fuzzy inference systems have been very seldom used in connection with investment analysis and decision-making, and this research is the first to present the use of a FIS together with the fuzzy pay-off method.

\section{THE PROPOSED NEW PROCEDURE FOR SUPPORTING INVESTMENT ANALYSIS WITH THE PAY-OFF METHOD}

Next, we present the proposed new procedure for supporting investment analysis with the pay-off method. A "starting" assumption is that there is an investment analysis model that uses the fuzzy pay-off method that has been constructed for 
the profitability analysis of the studied investment, and that is able to create a pay-off distribution of the investment profitability. Typically we expect that the model in place is akin to models typically used with the pay-off method, see, e.g., (Collan, 2012), and that it is able to consider the effect of important-to-profitability variables separately. The procedure is visually presented in Figure 2 .

For simplicity we have divided the proposed new procedure into four main steps:

Step 1. Identification of key investment variables $\tilde{V}_{i}$ and the range of their possible values

In the first step, key variables, $\tilde{V}_{i}$ (where $i=1, \cdots, n$, and $n$ is a number of variables) that are critical to the project profitability are identified. If the model is built such that these key variables are separately "already" identified, then the identification is very simple. An example of a key investment variable might, for example, be the quality of the main produced goods that affects the success of the product on the markets and that the company manufacturing the good can affect by quality management.

For each key variable the range of possible values is defined. The range can typically be identified by either addressing historical data, or by resorting to expert estimation. This range-determination is very similar to what is commonly done, when possible variable value ranges are determined for Monte Carlo simulation.

Step2. Identification and determination of relevant states $\widetilde{\mathrm{S}}_{\mathrm{ij}}$ for the identified key variables 
In the second step, the relevant states $\tilde{S}_{i j}$ (where $j=1, \cdots, N_{i}$, and $N_{i}$ is a number of relevant states of variable $\widetilde{V}_{\mathrm{i}}$ ) are identified for each key investment variable $\widetilde{V}_{i}$ that the investment management can affect. Relevant here means that the identified states are "clearly separate states" with regards to, e.g., the level of achievement they represent for the investment owners. This achievement may be, e.g., in terms of the above discussed quality and such that for quality four relevant states can be identified: "low", "medium", "good", and "excellent" quality. For each identified state the possible values within each state are recognized and the boundaries for each state are identified. If the states are fuzzy the exact representation for each state is determined. Variables that the investment management can affect means that if the management cannot affect, e.g., the raw material prices, then such variables need not (and cannot) be "achieved" by the investment owners. 


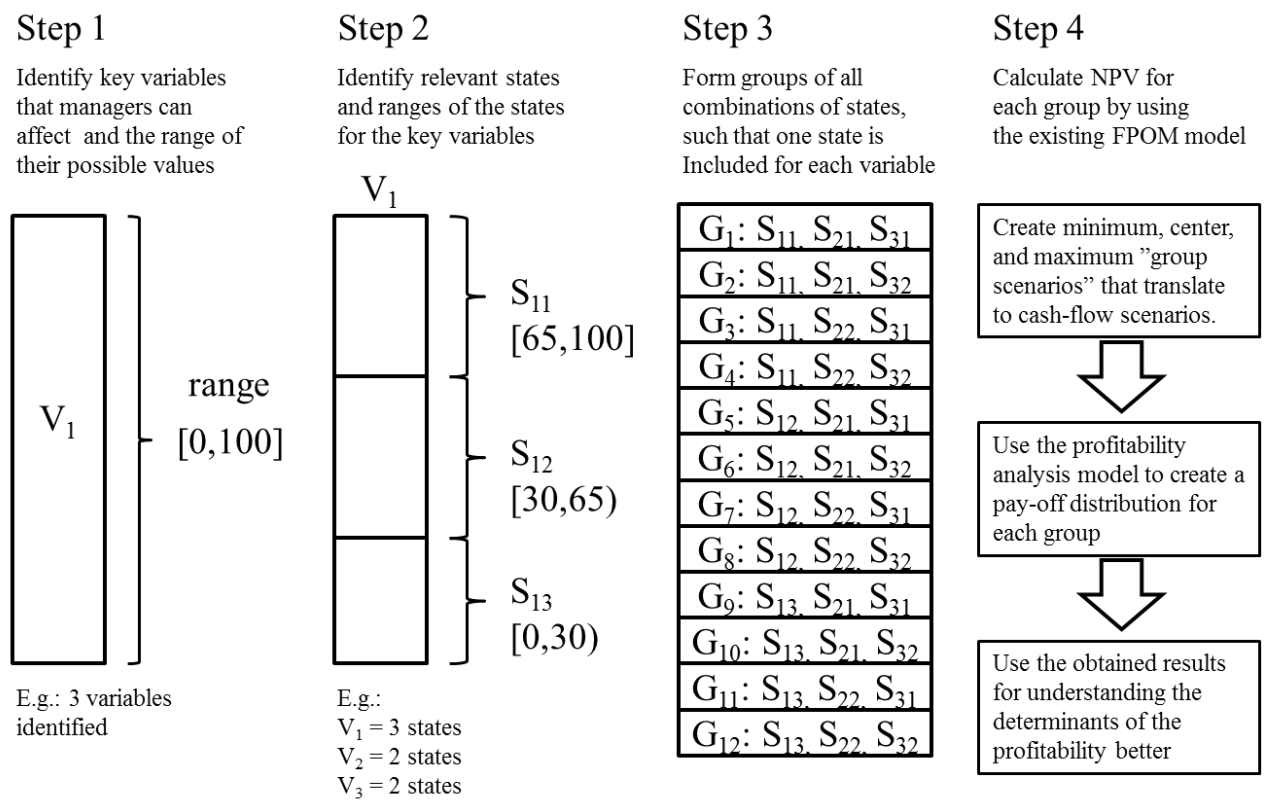

Figure 2: Presentation of the steps of the proposed new procedure

Step 3. Formation of groups $\widetilde{\mathrm{G}}_{\mathrm{k}}$ from all the possible combinations of the identified relevant states for the key variables, such that one state is included for all key variables

In the third step, groups $\tilde{G}_{k}$ (where $k=1, \cdots, K$ ) of all possible combinations that combine the identified states for (all) the key variables (simultaneously) are formed. Each one of the combinations is a "real-world" situation that may take place, e.g., such that three identified key variables states are quality = "low", marketing effort = "medium", and packaging = "excellent" and their combination is "low-medium-excellent". This grouping is the basis of the fourth step in the proposed procedure and also forms the premise part of the if-then rules in the FIS ('IF premise THEN consequent'). The number of rules (K) in the FIS rule base 
is determined by the number of state combinations, if all possible state combinations are considered so that one state is for each variable, then total amount of groups formed in this step is $K=\prod_{i=1}^{n} N_{i}$.

Step 4. Calculation of the fuzzy NPV for each group by applying the existing FPOM model

In the fourth step, the existing profitability analysis model is used to (separately) calculate the profitability (NPV) for each resulting group.

The step seems rather simple, but in fact includes making the choice of "how" the profitability is calculated for each resulting group. We suggest that for each group (combination) a set of three "scenarios" are identified that represent the "minimum", "center", and "maximum" values for the group. The values for the "minimum" and the "maximum" scenarios would come from the boundary values of the states in the group, while the "center" scenario value would either managerially set, or simply constructed from the mean values of the states that form the group. The three scenario values for each group would then be used in derivation of three cash-flow scenarios for the investment and ultimately the creation of the pay-off distribution for the investment under the circumstances (state combination) that the group represents. This means that $\mathrm{K}$ pay-off distributions are created.

We note that it is important to distinguish between "cost" and the "benefit" factors and to take care, when these are combined, while the group scenarios are constructed (Collan, 2012). 
Step 5. Defuzzification to real option value

$\mathrm{K}$ pay-off distributions are further aggregated into a single pay-off distribution based on crisp inputs. This aggregated distribution is automatically defuzzified into crisp expected NPV value. Alternatively, the real option value can be computed as the center of gravity of the positive side of the aggregated pay-off distribution weighted on the success ratio (Kozlova, Luukka, \& Collan, 2016).

By using the five above-mentioned steps, one is able to produce $\mathrm{K}$ pay-off distributions for the investment that represent the different circumstances that each group represents. Such sub-divided information, in addition to the "original" pay-off distribution, we feel, is able to offer a more detailed insight into the "mechanism" that has caused each profitability outcome. Because the variables selected are such that the management of the investment can act on them and can therefore "affect" the state in which the investment finds itself, the effect of any activities to move from one state to another may be studied. Typically such "movement" entails costs - having information on a detailed level about the effect of different states allows a meaningful discussion on the cost - benefit issue with regards to moving between states. The possibility to obtain the output $\mathrm{RO}$ value creates space for the RO valuation logic in the investment appraisal.

\section{CONSTRUCT OF A FUZZY INFERENCE SYSTEM FOR SUPPORTING INVESTMENT DECISION-MAKING}

Based on the above described new procedure for supporting investment analysis with the fuzzy pay-off method, and based on the observation that the structure of 
the method resembles the structure of an inference system, we have gone forward in this vein and propose a construct for an investment decision supporting fuzzy inference system. Building a FIS that formalizes the rules and assumes an inference mechanism to remain unchanged may sound at first a difficult match to the problem of investment decision-making, however, there are situations, where (a great) number of similar projects, within a similar reality, must be controlled for "fit" and evaluated within a tight timeframe (and where a formalized system would introduce structure and speed to the process). Such situations may, e.g., include the typically annually or semi-annually recurring analyses of patent portfolios (Camus \& Brancaleon, 2003; Carlsson, Fullér, Heikkilä, \& Majlender, 2007; Collan et al., 2013) and milestone reviews of research and development project portfolios (Collan \& Luukka, 2014; Hassanzadeh et al., 2012b; Karsak, 2006).

Also, at least two types of decision-making profiles can be identified that can use an inference system for support, we call these "policy making" and "investment decision-making". Policy makers can use a FIS to set thresholds, for what can be called policy-variables, and investors can use a FIS to aid in the investment decision-making.

On this background, we propose and present a MISO type fuzzy system, based on a Mamdani inference mechanism (Zadeh, 1973) for supporting investment decision-making. The rule base used consists of a "multiple input - one output"type of inference of the form:

$R_{1}: \quad$ If $\widetilde{\mathrm{V}}_{1} \mathrm{i} \widetilde{\mathrm{A}}_{11}$ and $\widetilde{\mathrm{V}}_{2}$ is $\widetilde{\mathrm{A}}_{21}$ and,$\ldots$, and $\widetilde{\mathrm{V}}_{\mathrm{n}}$ is $\widetilde{\mathrm{A}}_{\mathrm{n} 1}$, then $w$ is $N P V_{1}$ 
Rk: If $\widetilde{V}_{1}$ is $\widetilde{A}_{1 K}$ and $\widetilde{V}_{2}$ is $\widetilde{A}_{2 K}$ and, .., and $\widetilde{V}_{n}$ is $\widetilde{S}_{n K}$, then $w$ is $N P V_{K}$

fact: $\quad \widetilde{\mathrm{V}}_{1}$ is $\bar{v}_{01}$ and $\widetilde{\mathrm{V}}_{2}$ is $\bar{v}_{02}$ and,$\ldots$, and $\widetilde{\mathrm{V}}_{\mathrm{n}}$ is $\bar{v}_{0 n}$

consequence: $w$ is the NPV

where $\widetilde{\mathrm{V}}_{1}, \widetilde{\mathrm{V}}_{2}, \ldots, \widetilde{\mathrm{V}}_{\mathrm{n}}$ are the key variables, $\widetilde{\mathrm{A}}_{\mathrm{ij}}$ is the state for the $\mathrm{i}^{\text {th }}$ variable for the $\mathrm{k}^{\text {th }}$ rule. $N P V_{k}$ is the fuzzy NPV distribution for each group (see step 4 from the procedure presented in the previous section).

The consequence is computed by

$$
\text { consequence }=\operatorname{Agg}\left(\text { fact } \circ R_{1}, \ldots, \text { fact } \circ R_{K}\right)
$$

and the computation of the fuzzy output can be formulated as

i) Compute firing level of the k-th rule by $\widetilde{\mathrm{V}}_{1}\left(\bar{v}_{01}\right) \times \widetilde{\mathrm{V}}_{2}\left(\bar{v}_{02}\right) \times$ $, \ldots, \times \widetilde{\mathrm{V}}_{\mathrm{n}}\left(\bar{v}_{0 n}\right)$

ii) Compute the output of the k-th rule by

$$
N P V^{\prime}{ }_{k}(w)=\widetilde{V}_{1}\left(\bar{v}_{01}\right) \times \widetilde{V}_{2}\left(\bar{v}_{02}\right) \times, \ldots, \times \widetilde{V}_{\mathrm{n}}\left(\bar{v}_{0 n}\right) \rightarrow N P V_{k}(w)
$$

iii) Overall system output NPV is computed from individual rule outputs $\quad N P V^{\prime}{ }_{k}(w)$ by $\quad N P V(w)=$ $A g g\left\{N P V^{\prime}{ }_{1}, \ldots, N P V^{\prime}{ }_{K}\right\} \forall w \in W$

where $\rightarrow$ denotes the suitable implication operator, Agg, aggregation operator, and $\times$ the Cartesian product. We are applying a Mamdani inference mechanism, where firing levels of the rules are computed as: 


$$
\alpha_{k}=\widetilde{\mathrm{V}}_{1}\left(\bar{v}_{01}\right) \wedge \widetilde{\mathrm{V}}_{2}\left(\bar{v}_{02}\right) \wedge, \ldots, \wedge \widetilde{\mathrm{V}}_{\mathrm{n}}\left(\bar{v}_{0 n}\right)
$$

where $\wedge$ denotes the standard fuzzy intersection operator (minimum). The individual outputs from the rules are computed by:

$$
N P V^{\prime}{ }_{k}(w)=\alpha_{k} \wedge N P V_{k}(w)
$$

where $\wedge$ is Mamdani's implication (minimum). The overall output is computed by:

$$
N P V(w)=N P V^{\prime}{ }_{1}(w) \vee N P V^{\prime}{ }_{2}(w) \vee \cdots \vee N P V^{\prime}{ }_{K}(w)
$$

where for aggregation operator $v$ denotes the standard fuzzy union (maximum). As a last step for computing the single number defuzzified value, we apply the center of gravity operator for both, NPV (for further surface representation of results) and $\mathrm{RO}$ value (for real option analysis):

$$
\begin{gathered}
N P V_{0}=\frac{\int_{w} w N P V(w) d w}{\int_{w} N P V(w) d w} \\
R O V_{0}=\frac{\int_{w} w N P V^{+}(w) d w}{\int_{w} N P V^{+}(w) d w} \frac{\int_{w} N P V^{+}(w) d w}{\int_{w} N P V(w) d w}=\frac{\int_{w} w N P V^{+}(w) d w}{\int_{w} N P V(w) d w}
\end{gathered}
$$

Where $N P V^{+}(w)=\max \{0, N P V(w)\}$. The RO value computation is realized as a defuzzification operator for FIS and coded in Matlab® as follows 
function defuzzfun $=$ Rovdefuzz (xmf,ymf)

total_area $=\operatorname{sum}(y m f) ; \%$ the whole area of the fuzzy NPV

positive_area $=\operatorname{sum}(y m f(x m f>0)) ; \%$ positive area of the fuzzy NPV

mean_positive $=\operatorname{sum}(y m f(x m f>0) . * x m f(x m f>0)) /$ positive_area; $\%$ expected mean

success = positive_area/total_area; $\%$ success ratio

defuzzfun = mean_positive*success; \% real option value

end

\section{Published with MATLAB® R2015b}

The inference mechanism presented above is basic Mandani inference, which is now applying NPV or/and ROV computations. For the ROV computation the defuzzification operator needed to be implemented for Matlab as an add-on. In the following section we illustrate the proposed new procedure for investment analysis and the use of the proposed FIS in a real world investment context.

\section{NUMERICAL ILLUSTRATION: RENEWABLE ENERGY INVESTMENT}

This section presents a numerical illustration of using the proposed new investment analysis procedure in the analysis of a real-world renewable energy investment case in Russia. After illustrating the new investment analysis procedure, the use of a fuzzy inference system in decision-making is numerically presented in the same context, from the points of view of policy makers and of investors.

\subsection{CASE DESCRIPTION}

The investment case that used as background is an industrial scale $10 \mathrm{MW}$ solar photovoltaic (PV) power plant that is planned to be built in Russia, and that falls within the scope of Russian renewable energy (RE) support mechanism for the wholesale energy market (Boute, 2012; Government of Russian Federation, 
2013). The plant is assumed to start operation in 2018 and it is assumed to have a twenty-year economic life. The first fifteen years of the economic life are expected to be supported by the Russian RE support mechanism. More details on similar investments can be found in (Kozlova \& Collan, 2016). The RE investment faces uncertainty that comes from uncertain electricity prices and from inflation.

The Russian RE support mechanism is a contract that provides remuneration for selected investment projects that are paid regularly for a period of up to 15 years, starting from the power plant commercialization. The remuneration level is defined as a variable-rate annuity and the mechanism is designed to provide a secure return on $R E$ investments, while taking into account project related costs, the expected revenue from electricity sales, changes in the inflation, and the interest rate on the markets. The support cash-flows are substantial and account typically for $50-95 \%$ of total project revenues, depending on the technology type used, year of the contract, and on some other issues (Kozlova \& Collan, 2016).

In order to motivate investors to act in the best interest of the "system", the Russian RE support payments depend on the performance of the projects before investment and during the project economic life. The performance measures considered are:

- Capital expenses (CapEx); The mechanism sets a limit on the level of CapEx that an investment can have to be able to participate in the competition for the supported contracts - the idea is to push down the investment costs. 
- Capacity factor. Capacity factor is an indicator of electricity production performance that shows the ratio of the actual production of electricity to the theoretical maximum possible production $(\mathrm{T})$. The mechanism sets the value for a multiplicator that is used in calculating the year-ahead support payment level, based on the factual capacity factor achieved (F) the previous year as:

$$
\text { Capacity factor (multiplicator, } \%)=\left\{\begin{array}{c}
100 \%, \text { if } F>0.75 T \\
80 \%, \text { if } 0.5 T<F \leq 0.75 T \\
0, \text { if } F \leq 0.5 T
\end{array}\right.
$$

- Localization. The mechanism requires projects to employ a target share of equipment manufactured locally in Russia. If the localization target is achieved, a project gets full support payments, otherwise they are "almost halved" for the whole contract period.

As can be seen from the three measures above, the Russian RE support mechanism places an investment project into a 'rule-based' environment. Being able to "fulfill" requirements has an effect on the investment project profitability.

\subsection{Profitability ANALYSIS WITH THE NEW PROPOSED PROCEDURE}

Starting point of the analysis is a profitability analysis model that has been built, and that uses the fuzzy pay-off method to calculate the investment profitability. The computational logic behind the model is based on the Russian RE support policy that is presented in detail in (Kozlova \& Collan, 2016). The output from the model is a triangular fuzzy pay-off (NPV) distribution. Project cash-flows are estimated and comprise from revenues from the electricity sales and from the 
support payments that come from the Russian RE support mechanism, project costs include the investment cost, operating costs, and taxes. Based on the model the proposed procedure is used according to the steps described above:

Step 1: Five key variables are identified for the investment, these are:

$$
\tilde{V}=\left\{\tilde{V}_{1}, \tilde{V}_{2}, \tilde{V}_{3}, \tilde{V}_{4}, \tilde{V}_{5}\right\}
$$

$=\{$ Electricity price, CPI, CapEx level, Localization, Capacity factor $\}$

Estimated ranges for the values of each key variable are shown in Table 1. It can be observed that the investment owner can affect three of key variables: the capital expenditure level, the capacity factor, and the localization requirement.

Table 1. Identified key variables for the investment and estimated ranges that the variables can take

\begin{tabular}{|c|c|c|}
\hline \multirow{2}{*}{ Factor } & \multicolumn{2}{|c|}{ Range of values } \\
\hline & Minimum & Maximum \\
\hline Electricity price, rub./MWh & 1000 & 3000 \\
\hline Consumer price index (CPI) (inflation) & 1.00 & 1.70 \\
\hline CapEx level (percent of the limit) & $80 \%$ & $150 \%$ \\
\hline Capacity factor (percent of the target) & $30 \%$ & $120 \%$ \\
\hline Localization requirement & Failed & Fulfilled \\
\hline
\end{tabular}

Step 2: Three out of five variables are such that can be affected by the management, and for these three relevant states are identified and the boundaries of the states are determined. The different states for each variable are: 
CapEx level $=\left\{\tilde{S}_{11}, \tilde{S}_{12}\right\}=\{$ Within limit $[80 \%, 100]$, Over the limit

$$
\begin{gathered}
(100 \%, 150 \%]\} \\
\text { Localization }=\left\{\tilde{S}_{21}, \tilde{S}_{22}\right\}=\{\text { Failed, Fulfilled }\}
\end{gathered}
$$

Capacity factor $=\left\{\tilde{S}_{31}, \tilde{S}_{32}, \tilde{S}_{33}\right\}=\{$ Low $(30 \%, 50 \%]$, Medium

$$
\text { ((50\%,75\%],High }(75 \%, 120 \%]\}
$$

The boundaries of the states are determined for each state of each variable, and they are also visible in (9), above.

Step 3: We form all different groups of the combinations of the possible states, such that each group consists of combinations that include one possible state for each relevant key variable. The groups are visible in Table 2.

\begin{tabular}{|c|c|c|c|}
\hline Group & CapEx level & Localization & Capacity factor \\
\hline 1 & Within the limit $[80 \%, 100 \%]$ & \multirow{2}{*}{ Fulfilled } & \multirow{4}{*}{ High $(75 \%, 120 \%]$} \\
\hline 2 & Over the limit $(100 \%, 150 \%]$ & & \\
\hline 3 & Within the limit $[80 \%, 100 \%]$ & \multirow{2}{*}{ Failed } & \\
\hline 4 & Over the limit $(100 \%, 150 \%$ & & \\
\hline 5 & Within the limit $[80 \%, 100 \%]$ & \multirow{2}{*}{ Fulfilled } & \multirow{4}{*}{ Medium $(50 \%, 75 \%]$} \\
\hline 6 & Over the limit $(100 \%, 150 \%]$ & & \\
\hline 7 & Within the limit $[80 \%, 100 \%]$ & \multirow{2}{*}{ Failed } & \\
\hline 8 & Over the limit $(100 \%, 150 \%]$ & & \\
\hline 9 & Within the limit $[80 \%, 100 \%]$ & \multirow{2}{*}{ Fulfilled } & \multirow{4}{*}{ Low $(30 \%, 50 \%]$} \\
\hline 10 & Over the limit $(100 \%, 150 \%]$ & & \\
\hline 11 & Within the limit $[80 \%, 100 \%]$ & \multirow{2}{*}{ Failed } & \\
\hline 12 & Over the limit $(100 \%, 150 \%]$ & & \\
\hline
\end{tabular}

Table 2.The twelve groups constructed of the possible combinations of the relevant states

The information about the groups is later also used in the formation of the premise part of the rules for the fuzzy inference system. 
Step 4: The profitability for each group is calculated separately by using the "underlying" investment profitability analysis model. For the calculation, the minimum, center, and maximum values are set for each group. This is achieved by setting the identified boundary values of each state to serve as the minimum and the maximum values for each state. The center value for each state (and thus group) is calculated as the average of the minimum and the maximum of each state's value, with the exception of the variables "capital expenditure" and "capacity factor". The minimum, center, and maximum value for each group is derived by combining the minimum, center, and maximum values for the state combination that makes the group. Table 3 shows the minimum, center, and maximum values for each state, and for $\mathrm{CPI}$ and the electricity price used in the calculation.

Table 3. Minimum, center, and maximum values for each state and for the CPI and Electricity price variables. Resulting NPV in billions of rubles also indicated for each group.

\begin{tabular}{|l|l|l|l|l|l|l|l|}
\hline Group & Scenario & $\begin{array}{l}\text { Electricity } \\
\text { price, } \\
\text { rub./MWh }\end{array}$ & CPI & CapEx & Localization & $\begin{array}{l}\text { Capacity } \\
\text { factor }\end{array}$ & $\begin{array}{l}\text { NPV, } \\
\text { bln. } \\
\text { rub. }\end{array}$ \\
\hline \multirow{3}{*}{1} & Maximum & 3000 & 1 & $80 \%$ & Fulfilled & $120 \%$ & 0.21 \\
\cline { 2 - 8 } & Center & 2000 & 1.35 & $100 \%$ & Fulfilled & $97.5 \%$ & 0.17 \\
\cline { 2 - 8 } & Minimum & 1000 & 1.7 & $100 \%$ & Fulfilled & $75.1 \%$ & 0.15 \\
\hline \multirow{3}{*}{2} & Maximum & 3000 & 1 & $101 \%$ & Fulfilled & $120 \%$ & 0.21 \\
\cline { 2 - 8 } & Center & 2000 & 1.35 & $130 \%$ & Fulfilled & $97.5 \%$ & -0.09 \\
\cline { 2 - 6 } & Minimum & 1000 & 1.7 & $150 \%$ & Fulfilled & $75.1 \%$ & -0.29 \\
\hline \multirow{3}{*}{3} & Maximum & 3000 & 1 & $80 \%$ & Failed & $120 \%$ & -0.26 \\
\cline { 2 - 8 } & Center & 2000 & 1.35 & $100 \%$ & Failed & $97.5 \%$ & -0.46 \\
\cline { 2 - 5 } & Minimum & 1000 & 1.7 & $100 \%$ & Failed & $75.1 \%$ & -0.52 \\
\hline \multirow{3}{*}{4} & Maximum & 3000 & 1 & $101 \%$ & Failed & $120 \%$ & -0.39 \\
\cline { 2 - 8 } & Center & 2000 & 1.35 & $130 \%$ & Failed & $97.5 \%$ & -0.73 \\
\cline { 2 - 4 } & Minimum & 1000 & 1.7 & $150 \%$ & Failed & $75.1 \%$ & -0.96 \\
\hline 5 & Maximum & 3000 & 1 & $80 \%$ & Fulfilled & $75 \%$ & -0.02 \\
\hline
\end{tabular}




\begin{tabular}{|c|c|c|c|c|c|c|c|}
\hline & Center & 2000 & 1.35 & $100 \%$ & Fulfilled & $62.5 \%$ & -0.08 \\
\hline & Minimum & 1000 & 1.7 & $100 \%$ & Fulfilled & $50.1 \%$ & -0.08 \\
\hline \multirow{3}{*}{6} & Maximum & 3000 & 1 & $101 \%$ & Fulfilled & $75 \%$ & -0.06 \\
\hline & Center & 2000 & 1.35 & $130 \%$ & Fulfilled & $62.5 \%$ & -0.34 \\
\hline & Minimum & 1000 & 1.7 & $150 \%$ & Fulfilled & $50.1 \%$ & -0.53 \\
\hline \multirow{3}{*}{7} & Maximum & 3000 & 1 & $80 \%$ & Failed & $75 \%$ & -0.42 \\
\hline & Center & 2000 & 1.35 & $100 \%$ & Failed & $62.5 \%$ & -0.60 \\
\hline & Minimum & 1000 & 1.7 & $100 \%$ & Failed & $50.1 \%$ & -0.63 \\
\hline \multirow{3}{*}{8} & Maximum & 3000 & 1 & $101 \%$ & Failed & $75 \%$ & -0.56 \\
\hline & Center & 2000 & 1.35 & $130 \%$ & Failed & $62.5 \%$ & -0.87 \\
\hline & Minimum & 1000 & 1.7 & $150 \%$ & Failed & $50.1 \%$ & -1.09 \\
\hline \multirow{3}{*}{9} & Maximum & 3000 & 1 & $80 \%$ & Fulfilled & $50 \%$ & -0.67 \\
\hline & Center & 2000 & 1.35 & $100 \%$ & Fulfilled & $40 \%$ & -0.91 \\
\hline & Minimum & 1000 & 1.7 & $100 \%$ & Fulfilled & $30 \%$ & -0.96 \\
\hline \multirow{3}{*}{10} & Maximum & 3000 & 1 & $101 \%$ & Fulfilled & $50 \%$ & -0.85 \\
\hline & Center & 2000 & 1.35 & $130 \%$ & Fulfilled & $40 \%$ & -1.20 \\
\hline & Minimum & 1000 & 1.7 & $150 \%$ & Fulfilled & $30 \%$ & -1.45 \\
\hline \multirow{3}{*}{11} & Maximum & 3000 & 1 & $80 \%$ & Failed & $50 \%$ & -0.74 \\
\hline & Center & 2000 & 1.35 & $100 \%$ & Failed & $40 \%$ & -1.00 \\
\hline & Minimum & 1000 & 1.7 & $100 \%$ & Failed & $30 \%$ & -1.06 \\
\hline \multirow{3}{*}{12} & Maximum & 3000 & 1 & $101 \%$ & Failed & $50 \%$ & -0.93 \\
\hline & Center & 2000 & 1.35 & $130 \%$ & Failed & $40 \%$ & -1.29 \\
\hline & Minimum & 1000 & 1.7 & $150 \%$ & Failed & $30 \%$ & -1.54 \\
\hline
\end{tabular}

The NPV calculation procedure for each scenario of each group can be expressed as (see details in Appendix 1):

$$
C F_{i}=Y_{E_{i}}\left(\widetilde{\mathrm{V}}_{1_{\mathrm{i}}}, \widetilde{\mathrm{V}}_{5_{\mathrm{i}}}\right)+Y_{S_{i}}\left(\widetilde{\mathrm{V}}_{1_{\mathrm{i}}}, \widetilde{\mathrm{V}}_{\mathrm{i}_{\mathrm{i}}}, \widetilde{\mathrm{V}}_{3_{\mathrm{i}}}, \widetilde{\mathrm{V}}_{4_{\mathrm{i}}}, \widetilde{\mathrm{V}}_{5_{\mathrm{i}}}\right)-\widetilde{\mathrm{V}}_{3_{\mathrm{i}}}-C_{1_{i}} * \widetilde{\mathrm{V}}_{2_{\mathrm{i}}}-C_{2_{i}}
$$

where

$i$ denotes a year index of the project lifetime,

$Y_{E}$ is function of revenues from the electricity sales,

$Y_{S}$ is a function of RE support payments,

$\widetilde{V}_{1}$ is the electricity price,

$\widetilde{\mathrm{V}}_{2}$ is the $\mathrm{CPI}$, 
$\widetilde{V}_{3}$ is CapEx,

$\widetilde{\mathrm{V}}_{4}$ is localization,

$\widetilde{V}_{5}$ is capacity factor,

$C_{1}$ is operating expenses (OpEx), and

$C_{2}$ is taxes.

The resulting NPV for each scenario of each group is visible in Table 3. Studying the resulting NPVs allows the investment owner to understand the effect each group (combination of states) has on the investment profitability. This becomes intuitive, when the resulting pay-off distributions are visualized, see Figure 3.

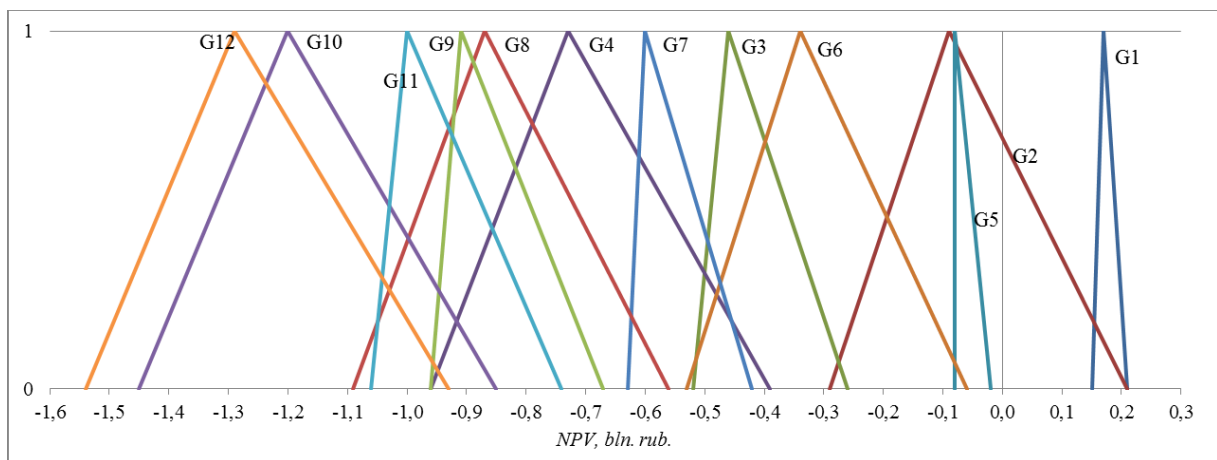

Figure 3: Visualization of the pay-off (NPV) distribution for each group

By visual inspection, a manager will find it easy to inspect which groups have possible outcomes on the positive NPV side. This information is "actionable" in the sense that it can be used to direct managerial actions in terms of reaching the necessary states of the relevant variables, so that the investment will reach 
profitability. If it can be immediately determined that the required states for a profitable outcome cannot be reached, the investment proposal can be abandoned.

\subsection{CONSTRUCTION OF A FIS FOR SUPPORTING INVESTMENT DECISION-MAKING}

Having the information we have about the connection between the groups and the NPV outcomes, we have what is needed to build the premise part for a rule base on Russian RE investment profitability. Each group that is a combination of variable states potentially corresponds to a premise part of a rule, in our case the total amount of potential rules is $K=\prod_{i=1}^{3} N_{i}=2 * 2 * 3=12$. To make sure the premise parts of the rules make sense, they are separately inspected for suitability - in our case, all twelve premise parts for the rules are accepted to be included in the rule base.

The formation of the premise part of the rule base is rather straightforward, for instance, the premise part of the rule number seven (according to the groups in Table 2) is 'IF CapEx is within the limit AND Localization is failed AND Capacity factor is medium'. After the premise part is formulated for each rule, the consequence part of each rule is formulated, in our case the consequence is the corresponding pay-off distribution. To create the complete rules, the premise and consequence parts are joined to create a set of twelve rules for the rule base:

1. IF CapEx is within the limit AND Localization is fulfilled AND Capacity Factor is high THEN NPV is \#1; 
2. IF CapEx is over the limit AND Localization is fulfilled AND Capacity Factor is high THEN NPV is \#2;

3. IF CapEx is within the limit AND Localization is failed AND Capacity Factor is high THEN NPV is \#3;

4. IF CapEx is over the limit AND Localization is failed AND Capacity Factor is high THEN NPV is \#4;

5. IF CapEx is within the limit AND Localization is fulfilled AND Capacity Factor is medium THEN NPV is \#5;

6. IF CapEx is over the limit AND Localization is fulfilled AND Capacity Factor is medium THEN NPV is \#6;

7. IF CapEx is within the limit AND Localization is failed AND Capacity Factor is medium THEN NPV is \#7;

8. IF CapEx is over the limit AND Localization is failed AND Capacity Factor is medium THEN NPV is \#8;

9. IF CapEx is within the limit AND Localization is fulfilled AND Capacity Factor is low THEN NPV is \#9;

10.IF CapEx is over the limit AND Localization is fulfilled AND Capacity Factor is low THEN NPV is \#10;

11.IF CapEx is within the limit AND Localization is failed AND Capacity Factor is low THEN NPV is \#11;

12.IF CapEx is over the limit AND Localization is failed AND Capacity Factor is low THEN NPV is \#12. 
The NPV consequences, expressed for each rule as triangular fuzzy numbers, can be seen in the right-most column in Table 3. Based on the rule base, a FIS that utilizes the rule base can be constructed. For the purposes of this research, we have identified two uses for (perspectives for the use of) the FIS: i) the use in policy-analysis, and ii) the use in investment decision-support. We further observe that the type of use affects the requirements that are set for the construct of the FIS.

The policy-analysis perspective is one, where the FIS is used to create and tune a policy. In this context the policy tool to be analyzed is the Russian renewable energy support mechanism, and it is tuned for the purposes of reaching the goals of the overall policy of renewable energy deployment. The policy analysis perspective typically means that clear boundaries for when, e.g., different levels of a support is paid are (and must for clarity be) established. When this requirement of non-ambiguity or "crispness" is taken into consideration, it means that a FIS designed to support policy-making will have crisp boundaries between different states that is, crisp boundaries between the input membership functions.

The investment decision support perspective is one, where the FIS is used to analyze the chances of a given investment to reach fulfillment of the set criteria, and as a result, of reaching the different observed levels of profitability. Each investment faces uncertainty and estimation imprecision with regards to the ability of the investor to reach a level of fulfillment of a criterion. This imprecision can be modeled by using fuzzy boundaries between the different states of the input membership functions of the FIS. 
Two versions of a FIS, with a policy-analysis and with an investment decisionsupport perspective, based on the created rule base, are presented in the following two sections.

\subsubsection{FIS with a policy-analysis perspective}

As discussed above, policy-analysis requires the setting of clear policies with a goal of shaping behavior in a desirable way. In the context of the Russian renewable energy support policy the policies require setting clear crisp thresholds between different $R E$ investment project specific factors that determine project performance that, in turn, determine the level of payments from the support mechanism. We model the membership functions of the fuzzy input variables with crisp boundaries between the states of the variables, and use trapezoidal fuzzy sets to model the states (for details see Appendix 2):

$$
\pi(x ; \alpha, \beta, \gamma, \delta)=\left\{\begin{array}{l}
\frac{x-\alpha}{\beta-\alpha} \text { if } \alpha \leq x \leq \beta \\
1 \text { if } \beta<x \leq \gamma \\
\frac{\delta-x}{\delta-\gamma} \text { if } \gamma<x \leq \delta \\
0 \text { otherwise }
\end{array}\right.
$$

As the thresholds are crisp we set $\alpha=\beta$ and $\gamma=\delta$. The resulting values for the states of the three support mechanism parameters with crisp borders are reported in Table 4. A visual representation of the crisp boundaries for the states of the three variables is visible in Appendix 2. 
Table 4. Values of each specified state of the three support mechanism parameters (policy-analysis perspective)

\begin{tabular}{|l|l|l|}
\hline Variable & State & $\begin{array}{l}\text { Trapezoidal fuzzy set } \\
(\alpha, \beta, \gamma, \delta)\end{array}$ \\
\hline CapEx level & $\begin{array}{l}\text { Within the limit }[80 \%, \\
100 \%]\end{array}$ & $(0.5,0.5,1,1)$ \\
\hline & $\begin{array}{l}\text { Over the limit }(100 \%, \\
150 \%]\end{array}$ & $(0,0,0.5,0.5)$ \\
\hline \multirow{5}{*}{ Localization } & Fulfilled & $(0.5,0.5,1,1)$ \\
\hline Capacity factor & Failed & $(0,0,0.5,0.5)$ \\
\hline & High $(75 \%, 120 \%]$ & $(0.3,0.3,0.5,0.5)$ \\
\hline & Medium $(50 \%, 75 \%]$ & $(0.5,0.5,0.75,0.75)$ \\
\hline & Low $(30 \%, 50 \%]$ & $(0.75,0.75,1.2,1.2)$ \\
\hline
\end{tabular}


Figure 4 shows a screenshot of the state combinations (rules) from the Matlab Fuzzy Logic Designer $\AA^{\circledR}$ interface, as well as the magnified output distribution. The vertical lines on the rules show a case with $\mathrm{CapEx}=0.4$, capacity factor $=$ 0.77 , and localization $=0.9$. As the input variable states are divided by crisp borders, the combinations are "unique" that is, any combination of crisp inputs will only fire one rule. This means that the resulting NPV is one of the twelve outcome NPVs "as is". In the case visualized in Figure 4 only rule \#2 fires.

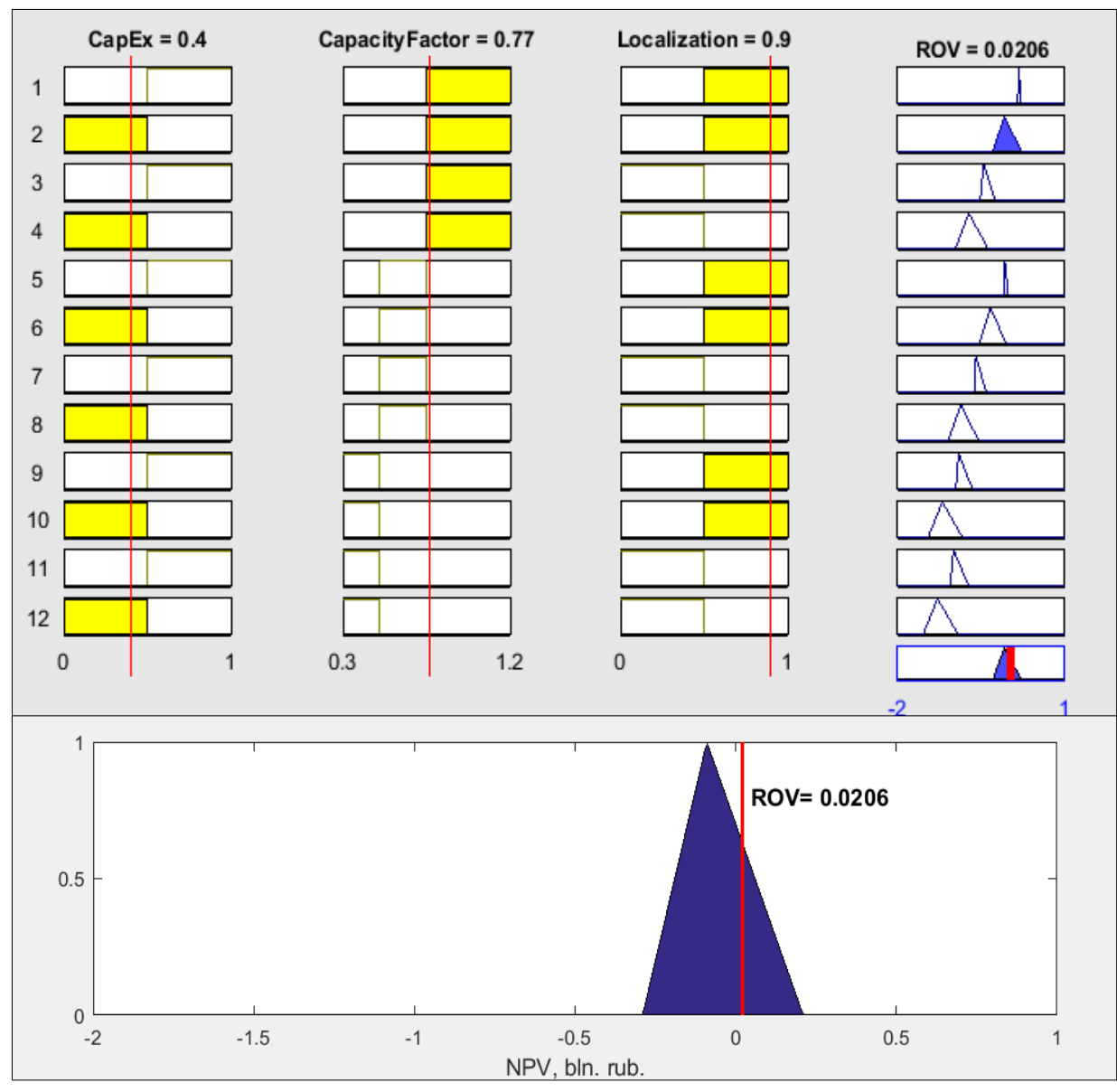

Figure 4. Fuzzy inference system visualization (policy-analysis perspective) 
For a better holistic view of the different profitability levels that are connected to the different rules, Figure 5 shows surface graphs of the expected NPV for the situations, where the localization variable is either in the fulfilled, or in the unfulfilled state, and the CapEx and the capacity factor variables' values are allowed to vary through their ranges.
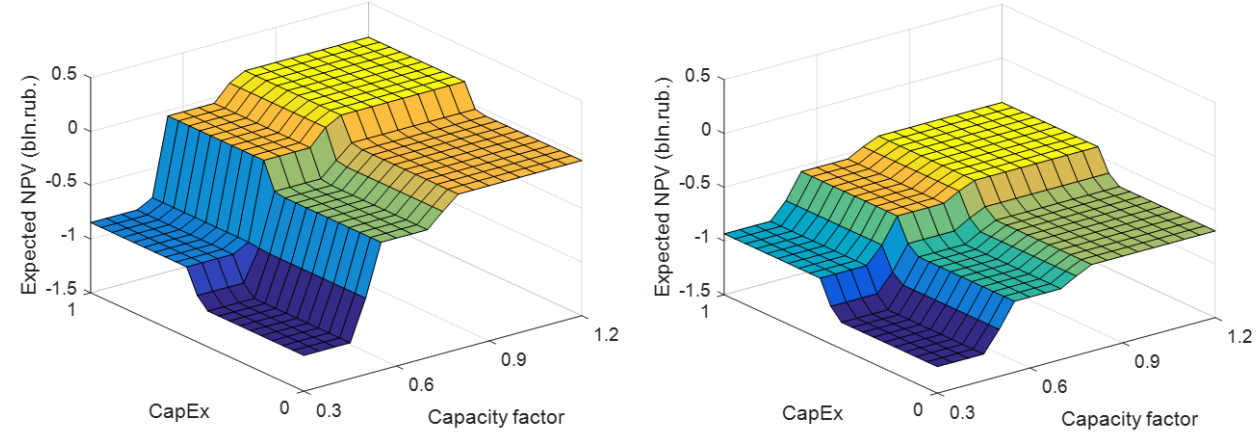

Figure 5. Expected NPV, when localization is fulfilled (left) / failed (right) and the other two variables vary through their full range.

In reality, localization is an issue that is more or less a requirement to even be able to participate in the project auctions, and for being an eligible recipient of the support offered by the Russian mechanism. These results show that taking the policy-maker perspective, with crisp boundaries of different states, clearly guides investments to a direction, where the CapEx is kept within the set limits, the capacity factor is kept high, and the localization requirement is fulfilled. Based on these results, the policy decision-makers can discuss, whether the boundaries are in the right place, or not.

\subsubsection{FIS with an investment decision-making perspective}


From the investing company's investment decision-making perspective, the focus is on "whether or not the company can fulfill the requirements" that is, whether the realized value at the time of establishing the support level is on the "right" side of the "variable state border", or not. This means that as there is uncertainty and imprecision in the ability of the managers to estimate the actual future outcome, the borders between states are imprecise and uncertain for the investor. This imprecision is reflected by modeling the membership functions of the input variable states as fuzzy. For the purposes of this research the states are modeled in a way that is presented in detail in Table 5.

Table 5. Values of each specified state of the three support mechanism parameters (investment decision-making perspective)

\begin{tabular}{|l|l|l|}
\hline Variable & State & Trapezoidal fuzzy set \\
\hline CapEx level & Within the limit $[80 \%$, & $(0,1,1,1)$ \\
$100 \%]$ & $\begin{array}{l}\text { Over the limit }(100 \%, \\
150 \%]\end{array}$ & $(0,0,0,1)$ \\
\cline { 2 - 3 } & Fulfilled & $(0,1,1,1)$ \\
\hline Localization & Failed & $(0,0,0,1)$ \\
\hline Capacity factor & High $(75 \%, 120 \%]$ & $(0.7,0.8,1.2,1.2)$ \\
\hline & Medium $(50 \%, 75 \%]$ & $(0.45,0.55,0.7,0.8)$ \\
\cline { 2 - 3 }$(30 \%, 50 \%]$ & $(0.30 .3,0.45,0.55)$ \\
\hline
\end{tabular}


A visual representation of the boundaries for the states of the three variables is visible in Appendix 2. Figure 6 shows a screenshot of the state combinations (rules), where the vertical line shows the same case as in the above example, with CapEx=0.4, capacity factor $=0.77$, and localization $=0.9$.

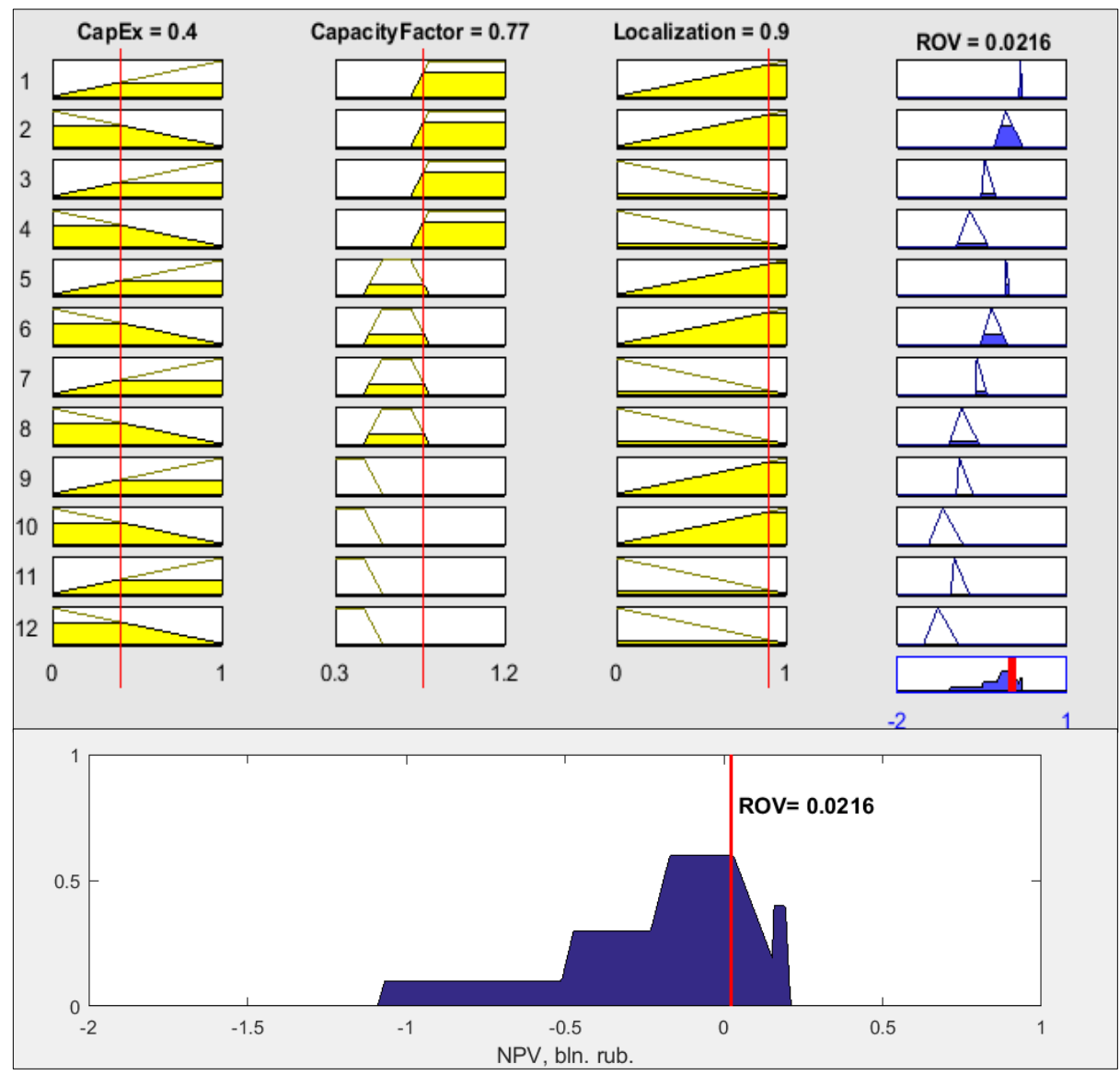

Figure 6. Fuzzy inference system visualization (investment analysis perspective)

The input variable states are divided by fuzzy borders and hence multiple rules are fired, in fact eight rules are fired. This means that also the outcome NPV is a 
construct, consisting of the outcomes of the eight fired rules, and in this case is a rather complex-looking NPV distribution. What can be seen from the magnified resulting NPV distribution is that the area of possible outcomes is rather wide, and exhibits the imprecision connected to the final outcome of the project.

The imprecision is reflected in Figure 7 that shows a surface graph of expected NPV, when the localization factor is either fulfilled, or not-fulfilled, by "smoothening" the graph considerably (compare to Figure 5). Distinct plains, or layers of similar profitability are not visible anymore. Uncertainty perceived by investors changes the overall picture, and in this case deteriorates the perception of the overall project profitability. A detailed two-dimensional representation of the results can be found in Appendix 3.
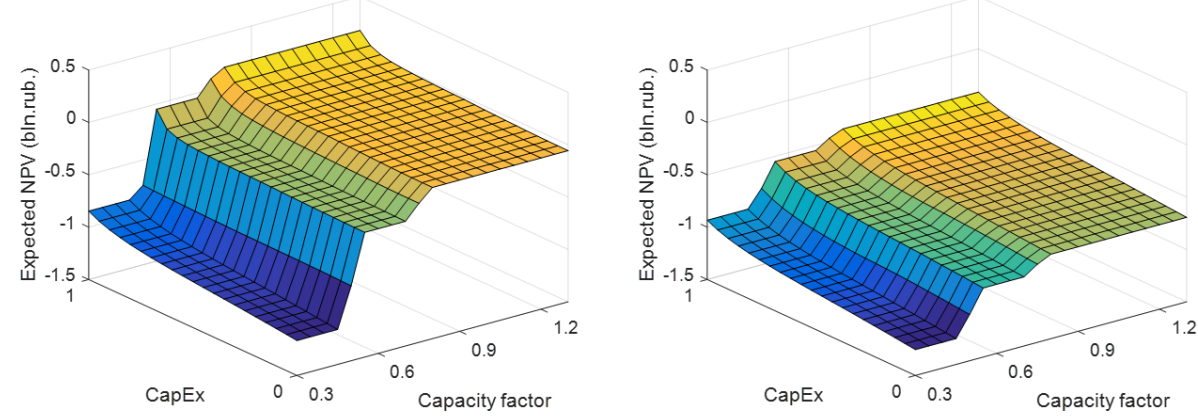

Figure 7. Expected NPV, when localization is fulfilled (left) / failed (right) and the other two variables vary through their full range.

What we can see from comparing the results from the two FIS, constructed for the policy analysis perspective and for the investment decision-making perspective, is that the different requirements for the uncertainty / imprecision 
that are incorporated in the FIS cause the results from these different FIS to be rather different. This observable difference can be understood as a quantification of how differently, in this context, the renewable energy support mechanism is viewed by investors and policy-makers. It seems that analysis of the FIS might be used in understanding the behavior of investors in light of different policies this kind of aspects are interesting, however, they fall outside the scope of this research.

\section{SUMMARY AND CONCLUSIONS}

This paper has introduced a new procedure for investment analysis and real option valuation to complement the typical profitability analysis performed with the fuzzy pay-off method. The idea of the procedure is to identify key variables for an investment that management of the investment can affect, and to find and to determine the boundaries of relevant "reachable" states for the value of these key variables. By using these relevant states as a basis, combinations of the states are formed that serve as scenarios of possible state-combination outcomes. Profitability is calculated for each state-combination, and by doing so, more information with regards to the cause-effect relationship between the statecombinations and profitability outcomes can be inferred. This information is actionable, as it can be used as a basis for decision-making with regards to actions that lead to reaching specific identified state combinations. Such information cannot be directly inferred from the typical analysis result from the fuzzy pay-off method. 
The identified state-combinations can be used also as a basis of constructing a rule base that serves as a component in a fuzzy inference system. The calculated profitability outcomes for each state-combination can act as the resultant part of a rule base. A construct of a fuzzy inference system in the context of the proposed new procedure for investment analysis was proposed.

The proposed new procedure and a FIS application into investment profitability analysis were numerically illustrated within the context of an investment into a renewable energy production investment in Russia. For the FIS illustration two separate perspectives that of a policy analysis, and that of investment decisionsupport, were taken. It was shown that there are notable differences between these perspectives, a finding that highlights the potential value in constructing FIS with different perspectives, for the purposes of better understanding the problems at hand and specifically investor behavior. More specifically, the two FIS show that different perceptions of uncertainty lead to different NPV profiles.

We believe that the proposed new method and the application of FIS into the investment profitability analysis context allow for better analysis of investments that face multiple key sources of uncertainty, and for which relevant states for these key uncertainties can be identified. Further research avenues include application of the proposed methods into other fields of industry and empirical testing of the proposed method with real world investments. 


\section{REFERENCES}

1. Ryan, P. A., Ryan, G. P. (2002). "Capital budgeting practices of the fortune 1000: How have things changed". Journal of Business and Management, Vol. 8, No. 4, p. 355-364.

2. Graham, J. R., Harvey, C. R. (2001). "The theory and practice of corporate finance: Evidence from the field". Journal of Financial Economics, Vol. 60, No. 2-3, p. 187-243.

3. Block, S. (2007). "Are "real options" actually used in the real world?". The Engineering Economist, Vol. 52, No. 3, p. 255-267.

4. Hacura, A., Jadamus-Hacura, M., Kocot, A. (2001). "Risk analysis in investment appraisal based on the monte carlo simulation technique". The European Physical Journal B-Condensed Matter and Complex Systems, Vol. 20, No. 4, p. 551-553.

5. Topal, E. (2008). "Evaluation of a mining project using discounted cash flow analysis, decision tree analysis, monte carlo simulation and real options using an example". International Journal of Mining and Mineral Engineering, Vol. 1, No. 1, p. 62-76.

6. Vithayasrichareon, P., MacGill, I. F. (2012). "A monte carlo based decision-support tool for assessing generation portfolios in future carbon constrained electricity industries". Energy Policy, Vol. 41, , p. 374-392.

7. Monjas-Barroso, M., Balibrea-Iniesta, J. (2013). "Valuation of projects for power generation with renewable energy: A comparative study based on real regulatory options". Energy Policy, Vol. 55, No. 0, p. 335-352.

8. Bastian-Pinto, C., Brandão, L., Hahn, W. J. (2009). "Flexibility as a source of value in the production of alternative fuels: The ethanol case". Energy Economics, Vol. 31, No. 3, p. 411-422.

9. Boomsma, T. K., Meade, N., Fleten, S. (2012). "Renewable energy investments under different support schemes: A real options approach". European Journal of Operational Research, Vol. 220, No. 1, p. 225-237.

10. Cortazar, G., Schwartz, E. S. (1998). "Monte carlo evaluation model of an undeveloped oil field". Journal of Energy Finance \& Development, Vol. 3, No. 1, p. $73-84$.

11. Zhu, L., Zhang, Z., Fan, Y. (2015). "Overseas oil investment projects under uncertainty: How to make informed decisions?". Journal of Policy Modeling, Vol. 37, No. 5, p. 742-762.

12. Kuchta, D. (2001). "A fuzzy model for R\&D project selection with benefit, outcome and resource interactions". The Engineering Economist, Vol. 46, No. 3, p. $164-180$.

13. Kuchta, D. (2000). "Fuzzy capital budgeting". Fuzzy Sets and Systems, Vol. 111, No. 3, p. 367-385.

14. Buckley, J. (1987). "The fuzzy mathematics of finance". Fuzzy Sets and Systems, Vol. 21, No. 3, p. 257-273.

15. Collan, M., Fullér, R., Mezei, J. (2009). "A fuzzy pay-off method for real option valuation". Journal of Applied Mathematics and Decision Sciences, Vol. 2009, p. 1-14.

16. Collan, M. (2012). "The pay-off method: Re-inventing investment analysis". CreateSpace Inc., Charleston, NC, USA, 
17. Kyläheiko, K. (1998). "Making sense of technology: Towards a synthesis between neoclassical and evolutionary approaches". International Journal of Production Economics, Vol. 56, , p. 319-332.

18. Collan, M., Haahtela, T., Kyläheiko, K. (2016). "On the usability of real option valuation model types under different types of uncertainty". International Journal of Business Innovation and Research, No. 11(1), p. 18-37.

19. Hassanzadeh, F., Collan, M., Modarres, M. (2012a). "A practical R\&D selection model using fuzzy pay-off method". The International Journal of Advanced Manufacturing Technology, Vol. 58, No. 1-4, p. 227-236.

20. Collan, M., Kinnunen, J. (2011). "A procedure for the rapid preacquisition screening of target companies using the pay-off method for real option valuation". リアルオプション研究, Vol. 4, No. 1, p. 117-141.

21. Collan, M., Heikkilä, M. (2011). "Enhancing patent valuation with the payoff method". Journal of Intellectual Property Rights, Vol. 16, No. 5, p. 377-384.

22. Kozlova, M., Collan, M., \& Luukka, P. (2015). Renewable energy in emerging economies: Shortly analyzing the Russian incentive mechanisms for renewable energy investments. International Research Conference "GSOM Emerging Markets Conference-2015: Business and Government Perspectives", Saint-Petersburg, Russia.

23. Collan, M. (2011). "Valuation of industrial giga-investments: Theory and practice". Fuzzy Economic Review, Vol. 16, No. 1, p. 21.

24. Bednyagin, D., Gnansounou, E. (2011). "Real options valuation of fusion energy R\&D programme". Energy Policy, Vol. 39, No. 1, p. 116-130.

25. Hassanzadeh, F., Collan, M., Modarres, M. (2012b). "A practical approach to R\&D portfolio selection using the fuzzy pay-off method". Fuzzy Systems, IEEE Transactions On, Vol. 20, No. 4, p. 615-622.

26. Kozlova, M., Collan, M., Luukka, P. (2016a). "Simulation decomposition: New approach for better simulation analysis of multi-variable investment projects". Fuzzy Economic Review, Vol. 21, No. 2, p. 3.

27. Brealey, R. A., Myers, S. C., Allen, F., \& Mohanty, P. (2012). Principles of corporate finance. Tata McGraw-Hill Education.

28. Borges, R., Meier, A. (2017). "COG-FPOM: Adapted fuzzy pay-off method for real options valuation". Internal Working Paper no 17-01, Université De Fribourg,

29. Luukka, P., Collan, M. (2015a). "New fuzzy insurance pricing method for giga-investment project insurance". Insurance: Mathematics and Economics, Vol. 65, , p. 22-29.

30. Vimpari, J., Junnila, S. (2015). "Theory of valuing building life-cycle investments". Building Research \& Information, , p. 1-13.

31. Vimpari, J., Kajander, J., Junnila, S. (2014). "Valuing flexibility in a retrofit investment". Journal of Corporate Real Estate, Vol. 16, No. 1, p. 3-21.

32. Collan, M., Fedrizzi, M., Luukka, P. (2013). "A multi-expert system for ranking patents: An approach based on fuzzy pay-off distributions and a TOPSIS-AHP framework". Expert Systems with Applications, Vol. 40, No. 12, p. 4749-4759. 
33. Collan, M., Kyläheiko, K. (2013). "Forward-looking valuation of strategic patent portfolios under structural uncertainty". Journal of Intellectual Property Rights, Vol. 18, No. 3, p. 230-241.

34. Luukka, P., Collan, M. (2015b). "New fuzzy insurance pricing method for giga-investment project insurance". Insurance: Mathematics and Economics, Vol. 65, , p. 22-29.

35. Collan, M., Fedrizzi, M., Luukka, P. (2016). "Possibilistic risk aversion in group decisions: Theory with application in the insurance of giga-investments valued through the fuzzy pay-off method". Soft Computing, , p. 1-12.

36. Favato, G., Cottingham, J., Isachenkova, N. (2015). "Blending scenarios into real options: Relevance of the pay-off method to management investment decisions". Journal of Finance and Accounting, Vol. 3, No. 2, p. 12-17.

37. Kozlova, M., Collan, M., Luukka, P. (2016b). "Comparison of the datarmathews method and the fuzzy pay-off method through numerical results". Advances in Decision Sciences, Vol. 2016,

38. Mamdani, E. H., Assilian, S. (1975). "An experiment in linguistic synthesis with a fuzzy logic controller". International Journal of Man-Machine Studies, Vol. 7, No. 1, p. 1-13.

39. Feng, G. (2006). "A survey on analysis and design of model-based fuzzy control systems". Fuzzy Systems, IEEE Transactions On, Vol. 14, No. 5, p. 676697.

40. Fazzolari, M., Alcala, R., Nojima, Y., Ishibuchi, H., Herrera, F. (2013). "A review of the application of multiobjective evolutionary fuzzy systems: Current status and further directions". Fuzzy Systems, IEEE Transactions On, Vol. 21, No. 1 , p. $45-65$.

41. Tanaka, K., Sano, M. (1994). "A robust stabilization problem of fuzzy control systems and its application to backing up control of a truck-trailer". Fuzzy Systems, IEEE Transactions On, Vol. 2, No. 2, p. 119-134

42. Chiou, C., Chiou, C., Chu, C., Lin, S. (2009). "The application of fuzzy control on energy saving for multi-unit room air-conditioners". Applied Thermal Engineering, Vol. 29, No. 2, p. 310-316.

43. CUI, J., TAN, J., AO, L., KANG, W. (2005). "Automatic focusing method based on fuzzy control and image processing technique [J]". Opto-Electronic Engineering, Vol. 6, p. 004.

44. Das, T., Kar, I. N. (2006). "Design and implementation of an adaptive fuzzy logic-based controller for wheeled mobile robots". Control Systems Technology, IEEE Transactions On, Vol. 14, No. 3, p. 501-510.

45. Al-zahra, K. A., Moosa, K., Jasim, B. H. (2015). "A comparative study of forecasting the electrical demand in Basra city using Box-Jenkins and modern intelligent techniques". Iraqi Journal for Electrical \& Electronic Engineering, Vol. 11 , No. 1

46. Aggarwal, S., Kumar, M., Saini, L., Kumar, A. (2011). "Short-term load forecasting in deregulated electricity markets using fuzzy approach". Journal of Engineering and Technology, Vol. 1, No. 1, p. 24.

47. Sachdeva, S., Singh, M., Singh, U., Arora, A. S. (2011). "Efficient load forecasting optimized by fuzzy programming and OFDM transmission". Advances in Fuzzy Systems, Vol. 2011, p. 2. 
48. Ying, L., Pan, M. (2008). "Using adaptive network based fuzzy inference system to forecast regional electricity loads". Energy Conversion and Management, Vol. 49, No. 2, p. 205-211.

49. Bacanli, U. G., Firat, M., Dikbas, F. (2009). "Adaptive neuro-fuzzy inference system for drought forecasting". Stochastic Environmental Research and Risk Assessment, Vol. 23, No. 8, p. 1143-1154.

50. Kazeminezhad, M., Etemad-Shahidi, A., Mousavi, S. (2005). "Application of fuzzy inference system in the prediction of wave parameters". Ocean Engineering, Vol. 32, No. 14, p. 1709-1725.

51. Mohandes, M., Rehman, S., Rahman, S. (2011). "Estimation of wind speed profile using adaptive neuro-fuzzy inference system (ANFIS)". Applied Energy, Vol. 88, No. 11, p. 4024-4032.

52. Esfahanipour, A., Aghamiri, W. (2010). "Adapted neuro-fuzzy inference system on indirect approach TSK fuzzy rule base for stock market analysis". Expert Systems with Applications, Vol. 37, No. 7, p. 4742-4748.

53. Chang, P., Liu, C. (2008). "A TSK type fuzzy rule based system for stock price prediction". Expert Systems with Applications, Vol. 34, No. 1, p. 135-144.

54. Patel, P. B., \& Marwala, T. (2006). Neural networks, fuzzy inference systems and adaptive-neuro fuzzy inference systems for financial decision making. Neural Information Processing, pp. 430-439.

55. Amindoust, A., Ahmed, S., Saghafinia, A., Bahreininejad, A. (2012). "Sustainable supplier selection: A ranking model based on fuzzy inference system". Applied Soft Computing, Vol. 12, No. 6, p. 1668-1677.

56. Oh, J., Yang, J., Lee, S. (2012). "Managing uncertainty to improve decision-making in NPD portfolio management with a fuzzy expert system". Expert Systems with Applications, Vol. 39, No. 10, p. 9868-9885.

57. Yang, M., Khan, F. I., Sadiq, R. (2011). "Prioritization of environmental issues in offshore oil and gas operations: A hybrid approach using fuzzy inference system and fuzzy analytic hierarchy process". Process Safety and Environmental Protection, Vol. 89, No. 1, p. 22-34.

58. Osiro, L., Lima-Junior, F. R., Carpinetti, L. C. R. (2014). "A fuzzy logic approach to supplier evaluation for development". International Journal of Production Economics, Vol. 153, p. 95-112.

59. Ganga, G. M. D., Carpinetti, L. C. R. (2011). "A fuzzy logic approach to supply chain performance management". International Journal of Production Economics, Vol. 134, No. 1, p. 177-187.

60. Oderanti, F. O., De Wilde, P. (2010). "Dynamics of business games with management of fuzzy rules for decision making". International Journal of Production Economics, Vol. 128, No. 1, p. 96-109.

61. Ustundag, A., Kılınç, M. S., Cevikcan, E. (2010). "Fuzzy rule-based system for the economic analysis of RFID investments". Expert Systems with Applications, Vol. 37, No. 7, p. 5300-5306.

62. Arasteh, A., Aliahmadi, A. (2014). "A proposed real options method for assessing investments". The International Journal of Advanced Manufacturing Technology, Vol. 70, No. 5-8, p. 1377-1393. 
63. Kozlova, M., Luukka, P., Collan, M. (2016). "Fuzzy inference system for real option valuation with the fuzzy pay-off method". Proceedings of NSAIS16, , p. 23-25.

64. Camus, C., Brancaleon, R. (2003). "Intellectual assets management: From patents to knowledge". World Patent Information, Vol. 25, No. 2, p. 155159.

65. Carlsson, C., Fullér, R., Heikkilä, M., Majlender, P. (2007). "A fuzzy approach to R\&D project portfolio selection". International Journal of Approximate Reasoning, Vol. 44, No. 2, p. 93-105.

66. Karsak, E. E. (2006). A generalized fuzzy optimization framework for R\&D project selection using real options valuation. Computational science and its applications-ICCSA 2006 (pp. 918-927) Springer.

67. Collan, M., Luukka, P. (2014). "Evaluating R\&D projects as investments by using an overall ranking from four new fuzzy similarity measure-based TOPSIS variants". Fuzzy Systems, IEEE Transactions On, Vol. 22, No. 3, p. 505-515.

68. Zadeh, L. A. (1973). "Outline of a new approach to the analysis of complex systems and decision processes". Systems, Man and Cybernetics, IEEE Transactions On, No. 1, p. 28-44.

69. Government of Russian Federation. (2013). 28 may 2013 Decree \#449 on the Mechanism of Promoting the use of Renewable Energy in the Wholesale Market of Electric Energy and Power, (2013).

70. Boute, A. (2012). "Promoting renewable energy through capacity markets: An analysis of the Russian support scheme". Energy Policy, Vol. 46, No. 0, p. 68-77.

71. Kozlova, M., Collan, M. (2016). "Modeling the effects of the new russian capacity mechanism on renewable energy investments". Energy Policy, Vol. 95, p. 350-360. 


\section{APPENDIX 1. Specifications of cash-flow calculation}

Here we provide the logic behind the cash-flow calculation performed in the investment model. It should be noted that for the purpose of easy perception, expressions are given in a simplified form missing some details. All key variables are denoted by $\widetilde{\mathrm{V}}$, whereas rest factors, which are assumed not to represent uncertainty to the model playing minor role, are denoted as $C$.

The overall expression for the cash flow calculation is:

$$
C F_{i}=Y_{E i}\left(\widetilde{V}_{1_{\mathrm{i}}}, \widetilde{\mathrm{V}}_{5_{\mathrm{i}}}\right)+Y_{S_{i}}\left(\widetilde{\mathrm{V}}_{1_{\mathrm{i}}}, \widetilde{\mathrm{V}}_{\mathrm{i}_{\mathrm{i}}}, \widetilde{\mathrm{V}}_{3_{\mathrm{i}}}, \widetilde{\mathrm{V}}_{4_{\mathrm{i}}}, \widetilde{\mathrm{V}}_{5_{\mathrm{i}}}\right)-\widetilde{\mathrm{V}}_{3_{\mathrm{i}}}-C_{1_{i}} * \widetilde{\mathrm{V}}_{2_{\mathrm{i}}}-C_{2_{i}}
$$

where

$i$ denotes an year index of the project lifetime, for simplicity, further we omit it, meaning that all calculation is performed for a particular year of the project,

$Y_{E}$ is function of revenues from electricity sales,

$Y_{S}$ is a function of subsidy payments,

$\widetilde{V}_{1}$ is electricity price,

$\widetilde{\mathrm{V}}_{2}$ is $\mathrm{CPI}$ (inflation),

$\widetilde{V}_{3}$ is capital costs (CapEx),

$\widetilde{\mathrm{V}}_{4}$ is localization,

$\widetilde{V}_{5}$ is capacity factor,

$C_{1}$ is operating expenses (OpEx), 
$C_{2}$ is taxes (that although depend on the rest of cash flows their derivation is constant and defined by the Tax Code).

The calculation of cash-flow consist of estimating project revenues from both, electricity sales and the subsidy payments, and from the expense-side, including capital expenses, operating expenses, and taxes.

Further we provide clarification of functions:

$Y_{E}-$ revenue from electricity sales

The revenue from electricity sales is simply the electricity price multiplied by the electricity production that is defined by capacity factor:

$$
Y_{E}=\widetilde{\mathrm{V}}_{1} \widetilde{\mathrm{V}}_{5} C_{3} C_{4},
$$

where

$C_{3}$ - number of hours per year, and

$C_{4}-$ installed capacity of the power plant.

1. $Y_{S}$ - subsidy payments

The amount of subsidy paid depends on all key variables and is computed in accordance with a formalized legislative procedure. In a nutshell, it represents all project expenses corrected on expected revenues from electricity sales and adjusted with several coefficients to provide proper motivation for investors. 


$$
Y_{S}=Y_{s h}\left(\widetilde{\mathrm{V}}_{1}, \widetilde{\mathrm{V}}_{2}\right) * Y_{l}\left(\widetilde{\mathrm{V}}_{5}\right) *\left(Y_{c}\left(\widetilde{\mathrm{V}}_{3}, \widetilde{\mathrm{V}}_{4}\right)+\widetilde{\mathrm{V}}_{2} C_{5}+C_{6}\right),
$$

where

$C_{5}$ is a norm of OPEX defined by the legislation,

$C_{6}$ is the expected property tax for the project,

$Y_{s h}$ is the share of expenses to be covered by the support, calculated as:

$$
Y_{s h}=1-\frac{\widetilde{\mathrm{V}}_{1} C_{3} C_{7}}{C_{8 \_a n n}+\widetilde{\mathrm{V}}_{2} C_{5}}
$$

where

the nominator of the ratio represents expected revenues from the electricity sales for an 'average project' with

$C_{7}$ - target capacity factor set by the legislation,

the denominator represents total expected 'average project'

expenses comprised of

$C_{8 \_a n n}-$ annualized weighted capital costs of projects that submitted bids for a particular commercialization year and technology, and

$C_{5}$ - inflated (with $\widetilde{\mathrm{V}}_{2}$ ) norm of project operating expenses, defined by the legislation. 
$Y_{l}$ is the load coefficient that reflects electricity production performance and is formed as follows:

$Y_{l}=\left\{\begin{array}{c}1, \quad \text { if } \widetilde{\mathrm{V}}_{5}>0.75 C_{7}\left({ }^{\prime} \text { High' }^{\prime} \text { state of capacity factor }\right) \\ 0.8, \text { if } 0.5 C_{7}<\widetilde{\mathrm{V}}_{5} \leq 0.75 C_{7}\left({ }^{\prime} \text { Medium' state of capacity factor }\right) \\ 0, \quad \text { if } \widetilde{\mathrm{V}}_{5} \leq 0.5 C_{7}\left(\text { (Low' }^{\prime} \text { state of capacity factor }\right)\end{array}\right.$

$Y_{c}$ is annualized project capital costs adjusted in accordance with the set limit $\left(C_{9}\right)$ and fulfilling localization in the following way:

$$
\begin{aligned}
& \widetilde{\mathrm{V}}_{3 \text { adj } 1}=\left\{\begin{array}{c}
\widetilde{\mathrm{V}}_{3}, \text { if } \widetilde{\mathrm{V}}_{3} \leq C_{9}\left({ }^{\prime} \text { Within the limit'state of CapEx }\right) \\
C_{9}, \text { if } \widetilde{\mathrm{V}}_{3}>C_{9}\left({ }^{\prime} \text { Over the limit'state of CapEx }\right)
\end{array}\right. \\
& \widetilde{\mathrm{V}}_{3_{\text {adj } 2}}=\left\{\begin{array}{c}
\widetilde{\mathrm{V}}_{3_{\text {adj } 1},} \text { if } \widetilde{\mathrm{V}}_{4}=1\left({ }^{\prime} \text { Fulfilled'state of localization }\right) \\
0, \quad \text { if } \widetilde{\mathrm{V}}_{4}=0\left({ }^{\prime} \text { Failed'state of localization }\right)
\end{array}\right. \\
& Y_{c}=\widetilde{\mathrm{V}}_{3 \text { adj2_ann }}, \\
& \text { where } \\
& \widetilde{\mathrm{V}}_{3 \text { adj2_ann }} \text { is annualized value of } \widetilde{\mathrm{V}}_{3 \text { adj2 }} \text {. }
\end{aligned}
$$


APPENDIX 2. Detailed boundary information for the input variables

Details on boundary formation of input variables, in particular Capital expenditures, localization, and capacity factor are presented in Figure A1 (policyanalysis perspective) and Figure A2 (investor-analysis perspective)
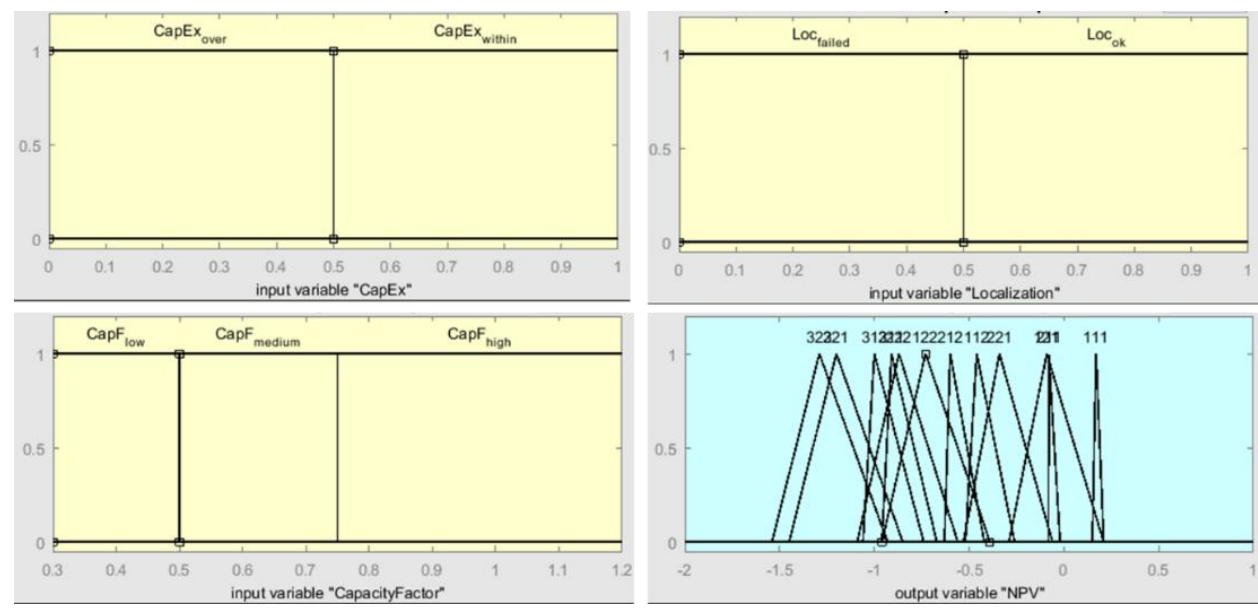

Figure A1. Variable states with crisp borders and the triangular NPV outcomes (policy-analysis perspective) 

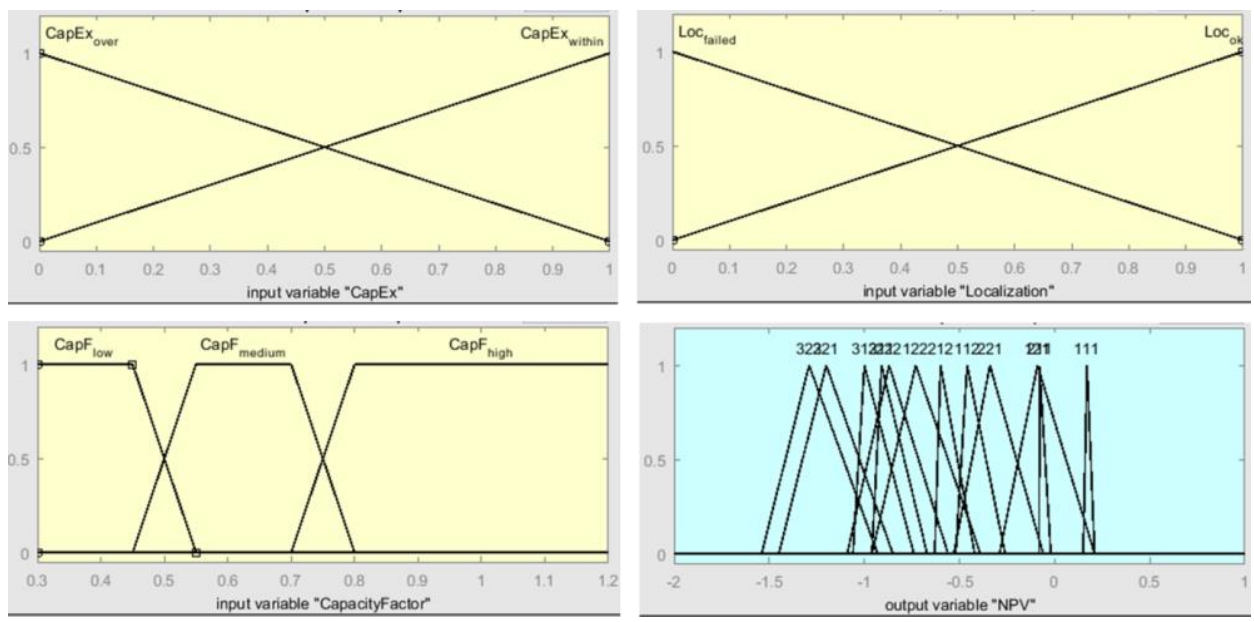

Figure A2. Variable states with imprecise borders and the triangular NPV outcomes (investor-analysis perspective) 


\section{APPENDIX 3. 2D representation of the results}

Here the results of examining our model by fixing two variable values (so that they are in 'profitable area') and representing another single variable value range. Results of this experiment from the policy-analysis perspective are shown in Table A1 and Figure A3 and from the investor-analysis perspective in Table A2 and Figure A4.

Table A1: Maximum NPVs when two variables have fixed values and one variable value is varied (policy-analysis perspective)

\begin{tabular}{|l|c|l|}
\hline Fixed variables & Best variable range & maximum NPV \\
\hline Capacity factor=1.2, & CapEx $\in[0.5,1]$ & 0.175 \\
Localization=1 & & \\
\hline CapEx=1,Localization=1 & Capacity factor & 0.175 \\
& $\in[0.76,1.2]$ & \\
\hline CapEx=1, Capacity factor $=1.2$ & Localization $\in[0.5,1]$ & 0.175 \\
\hline
\end{tabular}



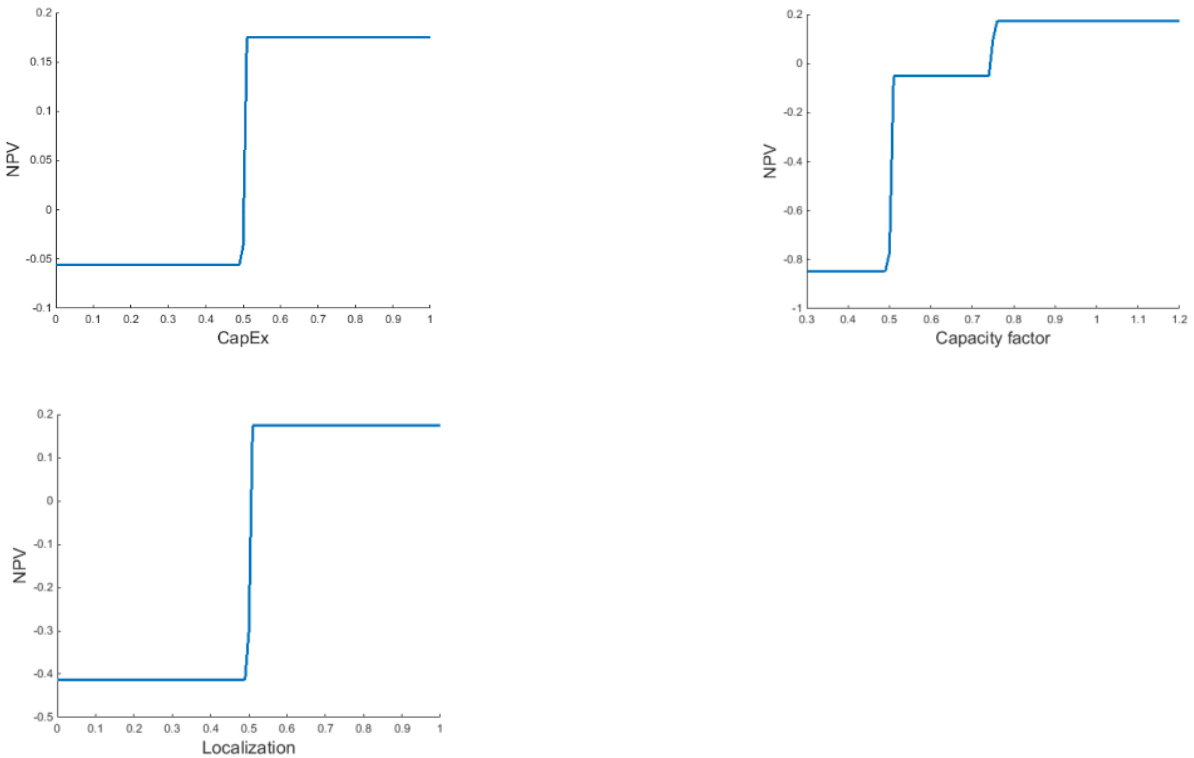

Figure A3: Change in NPV, when two variables are fixed according to Table 1, and one variable's values go through its entire range (policy-analysis perspective).

Table A2: Maximum NPVs when two variables have fixed values and one variable value is varied (investor-analysis perspective)

\begin{tabular}{|l|l|l|l|}
\hline Fixed variables & $\begin{array}{l}\text { Best variable } \\
\text { value }\end{array}$ & $\begin{array}{l}\text { Range for } \\
\text { NPV }>0\end{array}$ & $\begin{array}{l}\text { Maximum } \\
\text { NPV }\end{array}$ \\
\hline $\begin{array}{l}\text { Capacity factor=1.2, } \\
\text { Localization=1 }\end{array}$ & CapEx $=1$ & CapEx $\in[0.8,1]$ & 0.175 \\
\hline
\end{tabular}




\begin{tabular}{|l|l|l|l|}
\hline CapE $x=1$, Localization $=1$ & $\begin{array}{l}\text { Capacity factor } \\
\in[0.76,1.2]\end{array}$ & $\begin{array}{l}\text { Capacity factor } \\
\in[0.76,1.2]\end{array}$ & 0.175 \\
\hline Cap $E x=0, \quad$ Capacity & Localization & Localization & 0.175 \\
factor $=1.2$ & $=1$ & $\in[0.95,1]$ & \\
\hline
\end{tabular}
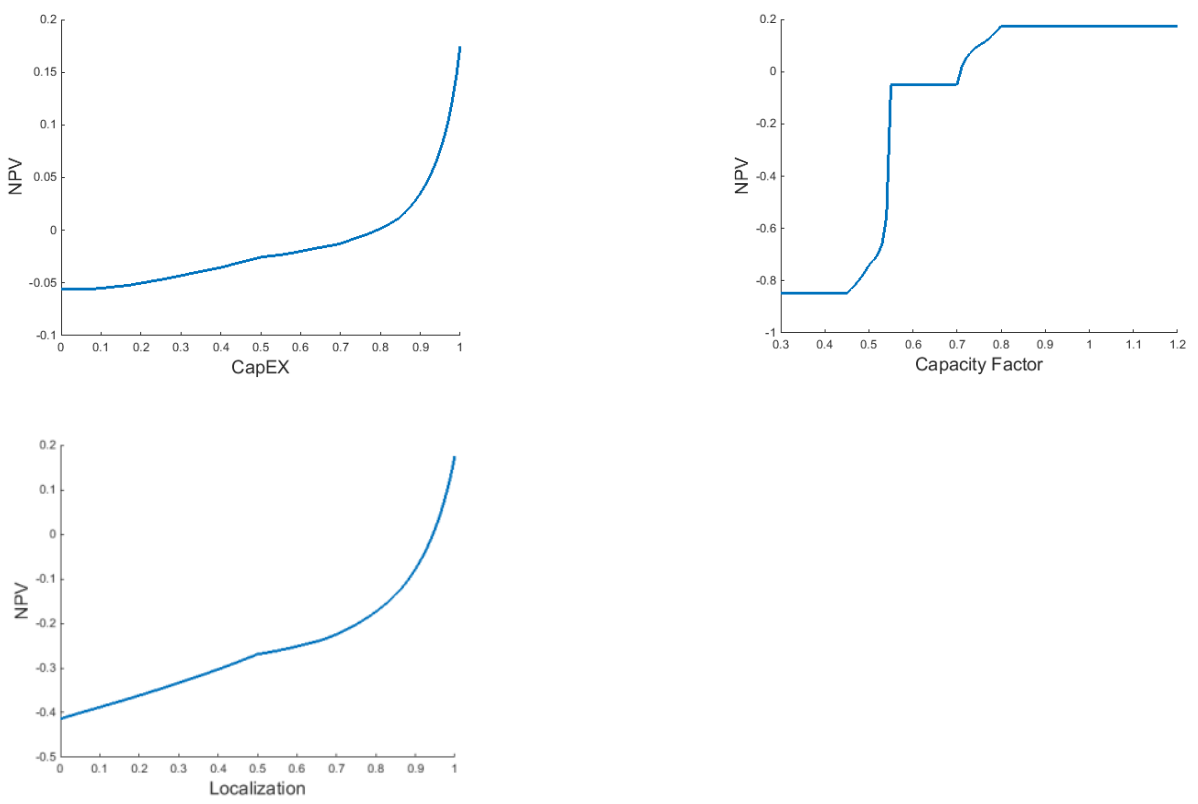

Figure A4: Change in NPV, when two variables are fixed according to Table 7, and one variable's values go through its entire range (investor-analysis perspective) 